\title{
Simulating the Galactic multi-messenger emissions with HERMES
}

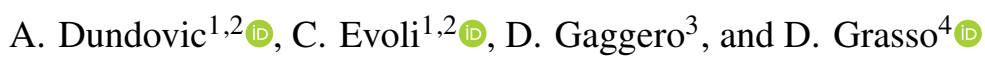 \\ 1 Gran Sasso Science Institute, Viale Francesco Crispi 7, 67100 L'Aquila, Italy \\ e-mail: carmelo.evoli@gssi.it \\ 2 INFN Laboratori Nazionali del Gran Sasso (LNGS), 67100 Assergi, L'Aquila, Italy \\ 3 Instituto de Física Teórica UAM-CSIC, Campus de Cantoblanco, 28049 Madrid, Spain \\ 4 INFN Sezione di Pisa, Polo Fibonacci, Largo B. Pontecorvo 3, 56127 Pisa, Italy
}

Received 12 March 2021 / Accepted 21 May 2021

\begin{abstract}
Context. The study of nonthermal processes such as synchrotron emission, inverse Compton scattering, bremsstrahlung, and pion production is crucial to understanding the properties of the Galactic cosmic-ray population, to shed light on their origin and confinement mechanisms, and to assess the significance of exotic signals possibly associated to new physics.

Aims. We present a public code called HERMES which is designed generate sky maps associated to a variety of multi-messenger and multi-wavelength radiative processes, spanning from the radio domain all the way up to high-energy gamma-ray and neutrino production.

Methods. We describe the physical processes under consideration, the code concept and structure, and the user interface, with particular focus on the python-based interactive mode. In particular, present the modular and flexible design that allows the user to easily extend the numerical package according to their needs.

Results. In order to demonstrate the capabilities of the code, we describe the details of a comprehensive set of sky maps and spectra associated to all physical processes included in the code. We comment in particular on the radio, gamma-ray, and neutrino maps, and mention the possibility of studying signals stemming from dark matter annihilation.

Conclusions. HERMES can be successfully applied to constrain the properties of the Galactic cosmic-ray population, improve our understanding of the diffuse Galactic radio, gamma-ray, and neutrino emission, and search for signals associated to particle dark matter annihilation or decay.
\end{abstract}

Key words. gamma rays: diffuse background - radio continuum: ISM - cosmic rays

\section{Introduction}

The Milky Way galaxy has been recognized over recent decades as a bright broad-band source of diffuse nonthermal multimessenger radiation, from $\mathrm{MHz}$ radio waves all the way up to multi-TeV gamma-rays and neutrinos. A coherent modeling of the different components that contribute to these emissions, and confrontation with available data, is required to reach a comprehensive understanding of the nature of this radiation.

In the low-frequency domain, the radio and microwave emissions trace several key interactions between the Galactic cosmic-ray (CR) leptons and the interstellar medium (ISM): in particular, the synchrotron component originates from interactions with the Galactic magnetic field. For magnetic field intensities of $O(1) \mu \mathrm{G}$, comparable with the typical values inferred in the Milky Way, and for CR leptons of [GeV-TeV] energies, the synchrotron radiation falls in the $[\mathrm{MHz}-\mathrm{GHz}]$ frequency range. Therefore, the study of the radio maps via extensive surveys, together with a variety of complementary observables - including, for instance, the deflection of ultra-high energy cosmic rays (UHECRs), and the study of the Faraday rotation of polarized sources - allows the distribution of highenergy CR leptons and the structure of the Galactic magnetic field to be constrained and modeled. This line of research has been developed over the last and current century and has led to the identification of large coherent magnetic structures in the Galaxy with ever improving accuracy (Beuermann et al.
1985; Men et al. 2008; Sun et al. 2008; Sun \& Reich 2010; Jaffe et al. 2010, 2011; Fauvet et al. 2011; Jansson \& Farrar 2012; Unger \& Farrar 2017; Hutschenreuter et al. 2021). As far as CR studies are concerned, the analysis of the Galactic synchrotron maps revealed useful information about the spectral properties of the diffuse population of relativistic leptons and their distribution in the Galactic halo (Strong et al. 2011; Di Bernardo et al. 2013). Within the same frequency range, freefree emission is produced by thermal electrons scattering off ions in the ionized component of the ISM. This process is particularly relevant above a few $\mathrm{GHz}$ in the Galactic plane in emission and below $\simeq 300 \mathrm{MHz}$ in absorption.

On the other end of the electromagnetic spectrum, gammaray and neutrino astronomy are younger disciplines. The first detection of a diffuse signal above $50 \mathrm{MeV}$ from the Galactic plane dates back to the pioneering observations performed by the OSO-3 Satellite in 1967-1968 (Kraushaar et al. 1972) followed by SAS-2 (Kniffen et al. 1973), COS-B (Lebrun et al. 1982), and EGRET (Hunter et al. 1997).

Over recent years, the AGILE telescope (Tavani et al. 2009) and the Fermi Gamma-Ray Space Observatory (Atwood et al. 2009) have enriched the catalog of Galactic and extra-galactic gamma-ray sources, also providing detailed full-sky maps of the diffuse emission up to $1 \mathrm{TeV}$ with an angular resolution reaching $\simeq 0.1^{\circ}$. At even larger energies, a crucial role is played by ground-based Atmospheric Cherenkov Telescopes (ACTs) such as H.E.S.S. (Aharonian \& H.E.S.S. Collaboration 2000), 
VERITAS (Weekes et al. 2002), MAGIC (Cortina 2005), and Water Cherenkov Telescopes (WCTs) such as MILAGRO (Sullivan \& MILAGRO Collaboration 2001) and HAWC (Westerhoff 2014). The ACTs feature a limited field of view, but they can probe the diffuse emission with higher sensitivity and resolution in limited but very compelling regions like the Galactic center (GC) or several star-forming regions.

Thanks to the accuracy of the current measurements provided by satellite experiments, new unexpected features have emerged in the spatial properties of the diffuse CR sea (Gaggero et al. 2015a; Acero et al. 2016; Yang et al. 2016; Pothast et al. 2018), with profound implications for the underlying micro-physics of CR transport (Cerri et al. 2017; Recchia et al. 2016), and phenomenological implications for the interpretation of very-high-energy gamma-ray and neutrino data (Gaggero et al. 2015b, 2017; Neronov \& Semikoz 2016; Pagliaroli et al. 2016; Cataldo et al. 2019). Moreover, groundbased experiments have revealed the first signs of high-energy diffuse emission from bright regions near the $\mathrm{GC}$ in the $\mathrm{TeV}$ domain.

At these energies, gamma-rays are partially attenuated because of the presence of the interstellar radiation fields, and therefore high-energy neutrinos offer a valuable complementary probe of the hadronic components of CRs. High-energy $(E \gtrsim 10 \mathrm{TeV})$ neutrino astronomy has recently come online with the detection of several neutrinos of unambiguous astrophysical origin by the IceCube observatory at the South Pole (see Ahlers \& Halzen 2018 for a recent review). Recently, the experiment ANTARES in the Mediterranean sea also joined the effort for the detection of astrophysical neutrinos (Albert et al. 2018a). In particular, a combined analysis of the Galactic plane performed by the two experiments provided the first hint of a component of the emission caused by the galactic CR population (Albert et al. 2017, 2018b).

Besides being a relevant problem perse, the study of the diffuse nonthermal emission from the Galaxy has an important link to the quest for the elusive dark matter (DM) component that permeates the Universe. Under the hypothesis that this substance is made of weakly interacting massive particles (WIMPs), a gamma-ray signal may be expected from the annihilation or the decay of those particles in the DM halo that embeds the Milky Way galaxy. The search for such a signal in DM-rich regions, in particular the inner Galaxy, is a challenging task that requires accurate modeling of the astrophysical processes and advanced data analysis, and is clearly hampered by the complexity of the astrophysical processes outlined above (see Gaggero \& Valli 2018 for a review).

The future is extremely promising for both extremities of the electromagnetic spectrum and for the neutrino channel, opening unprecedented scientific opportunities for the coming years. In the radio domain, the Square Kilometer Array (SKA) will operate over a wide range of frequencies, and its size will typically improve the sensitivity by a factor of $\sim 50$ compared to previous instruments (Weltman et al. 2020). In the very-high-energy gamma-ray domain, the Cherenkov Telescope Array (CTA; Cherenkov Telescope Array Consortium 2019) and the Large High Altitude Air Shower Observatory (LHAASO; Bai et al. 2019) will provide a giant leap in sensitivity in both the Norther and Southern hemisphere.

In particular, CTA will characterize high-energy emitters and the diffuse emission along the Galactic plane, helping to improve our understanding of $\mathrm{CR}$ acceleration up to PeV energies and of the properties of the $\mathrm{CR}$ diffuse sea above the TeV. It will also help in advancing the search for a DM signal from multi-TeV
WIMPs with an annihilation cross-section as low as the one usually associated to thermal production in the early Universe. Concerning neutrinos, IceCube (and future extensions) will soon be assisted by KM3NeT (Adrián-Martínez et al. 2016) which, being located in the Mediterranean sea, will allow a better and more accurate view of the inner Galactic plane.

The plethora of existing data discussed above and the impressive increase in sensitivity expected from future experimental facilities motivates a complementary effort on the theoretical side. Several public codes have been released over the years to model at least some of those data sets. Concerning the radio window, the HAMMURABI ${ }^{1}$ code was specifically designed to simulate the Galactic synchrotron emission and Faraday rotation (Waelkens et al. 2009). In particular, its latest release entails an accurate description of the turbulent field and the calculation of the polarized emission (Wang et al. 2020). In the gamma-ray range, GALPROP ${ }^{2}$ has been widely used to reproduce the general features of the interstellar gamma-ray emission over the whole sky (Strong et al. 2009; Moskalenko et al. 2019). GALPROP is designed to simulate Galactic CR propagation and associated diffuse emissions simultaneously. The code computes the gas-related gamma-ray intensities using the CR flux computed beforehand and the column densities of $\mathrm{HI}$ and $\mathrm{H} 2$ for Galactocentric annuli based on $21-\mathrm{cm}$ and CO surveys as described in Strong et al. (2004). The inverse Compton scattering is treated using the formalism for an anisotropic radiation field developed by Moskalenko \& Strong (2000). A new version of the code ${ }^{3}$ includes detailed calculation of the synchrotron (also in polarization) and free-free emission, and provides the possibility to compare different recent models of the Galactic magnetic field. A semi-analytical approach to modeling the main components of the diffuse gamma-ray emission of the Galaxy has also been proposed (Delahaye et al. 2011). The CLUMPY ${ }^{4}$ code provides a comprehensive framework with which to compute indirect gamma-ray and $v$ signals from the Galactic DM annihilation or decay extending to the extragalactic scales and to model the contribution from substructures (Hütten et al. 2019). The PICARD 5 code - which introduced a new and fast numerical treatment of cosmic-ray transport in 3D - was recently applied to model the diffuse gamma-ray emission of the Galaxy between $100 \mathrm{MeV}$ and $100 \mathrm{TeV}$ (Kissmann 2014; Kissmann et al. 2015). Finally, in the context of gamma-ray analyses, we mention the $\mathrm{D}^{3} \mathrm{PO}$ framework (Selig et al. 2015). This method is designed to remove the shot noise, deconvolve the instrumental response, and to provide estimates for the different flux components separately by means of a Bayesian inference technique.

Here, we present the simulation framework HERMES (HighEnergy Radiative MESsengers) which joins these efforts and can be used to constrain the properties of the Galactic CR population, increasing our understanding of the radio and gamma-ray diffuse Galactic emission.

HERMES is designed for efficient development of astrophysical predictions for Galactic diffuse emissions. Users can assemble modules of the relevant quantities describing the Galactic environments (magnetic fields, radiation fields, CR densities, etc.), including their own modules, and receive as an output full-sky radio, gamma-ray, or neutrino maps associated to the

\footnotetext{
https://github.com/hammurabi-dev/hammurabix

https://galprop.stanford.edu

https://gitlab.mpcdf.mpg.de/aws/galprop

https://gitlab.com/clumpy/CLUMPY

https://astro-staff.uibk. ac . at/ kissmrbu/Picard. html
} 
following nonthermal radiative processes: (a) radio emission due to free-free scattering of thermal electrons onto the ionized component of the ISM; (b) synchrotron emission in the $\mathrm{MHz}-\mathrm{GHz}$ domain (eventually including the absorption by freefree); (c) gamma-ray emission by Inverse Compton scattering of the diffuse low-energy background photons; (d) gammaray and neutrino emission by pion-decay (eventually including the gamma-ray absorption by pair production); (e) gamma-ray emission by bremsstrahlung; (f) and gamma-ray and neutrino emission by DM annihilations in the dark matter halo. We also implement the calculation of the Galactic faraday rotations and dispersion measures as they can be used to constrain the distribution of the magnetic fields and free electron density independently of the CR density.

From a technical point of view, HERMES features a modular $\mathrm{C}++$ structure combined with a Python interface for userfriendliness (specifically in the $\mathrm{I} / \mathrm{O}$ ). This combination takes advantage of the popularity of Python (Momcheva \& Tollerud 2015) and at the same time benefits from the fast computational performance of a C++ code (Portegies Zwart 2020).

The paper is structured as follows. In Sect. 2 we briefly summarize the physics of the processes involved. In Sect. 3, the code structure of the program is presented. The capabilities for simulating diffuse emissions are demonstrated in Sect. 4 in a few examples where we also introduce a selected choice of up-todate models of the CR distributions, Galactic magnetic fields, Galactic gas distribution (ionized, atomic, and molecular), and interstellar radiation fields (ISRFs) that are included in the program. Finally, results are summarized in Sect. 5.

The program source code is licensed under the GNU General Public License v3 and is publicly available ${ }^{6}$ together with installation instructions and examples ${ }^{7}$. Questions and comments can be submitted to the ticketing system ${ }^{8}$.

\section{Physical processes included in the code}

In this section, we describe the physical processes that contribute predominantly to the gamma-ray $\left(E_{\gamma} \gtrsim 100 \mathrm{MeV}\right)$ and radio $(v$ in $\mathrm{MHz} \div 100 \mathrm{GHz}$ ) diffuse emissions from our Galaxy and their implementation in the HERMES code.

The local emissivities (i.e., production rate per unit volume) are expressed as $\epsilon_{\mathrm{E}}$ or $\epsilon_{v}$ depending on whether the emissivity is expressed as differential in energy or in frequency, respectively.

The differential intensity (differential flux per unit angle) as a function of direction in the sky is obtained by integrating the emissivity over the line of sight (LOS) distance $s$ :

$I(x, l, b)=\frac{1}{4 \pi} \int_{0}^{\infty} \mathrm{d} s \epsilon_{x}(\boldsymbol{r}, x)$

where the longitude $l$ and the latitude $b$ constitute a spherical coordinate system centered on the Sun and $x$ is either the gamma-ray energy $E_{\gamma}$ or the radio frequency $v$.

The conversion between $(l, b, s)$ and the cartesian coordinate system centered at the Galactic center $\boldsymbol{r}=(x, y, z)$ is given by:

$z=s \sin b, \quad x=s \cos b \cos l-r_{\odot}, \quad y=s \cos b \sin l$.

In the code we adopt SI units.

\footnotetext{
6 https://github.com/cosmicrays/hermes

https://github.com/cosmicrays/hermes-examples

8 https://github.com/cosmicrays/hermes/issues
}

\subsection{Faraday rotation}

The polarization angle of an electromagnetic wave is rotated when crossing a magnetized plasma, an effect known as Faraday rotation (see e.g., Rybicki \& Lightman 1979). Faraday effects on pulsar and extragalactic radio source data have been used to model the magnetic fields of the Milky Way (Pshirkov et al. 2011). In particular, the Faraday rotation towards pulsars are due to the ISM in the direction of the inner Galaxy, while data on extragalactic sources provide information about the magnetic fields over much of the regions above and below the Galactic plane.

The rotation measure (RM) quantifies the rate of change of the polarization angle $\chi$ as expressed by the formula:

$\chi=\mathrm{RM} \lambda^{2}+\chi_{0}$,

where $\chi_{0}$ is the original (or intrinsic) angle at the polarized source and $\lambda$ is the observation wavelength.

The RM is given by the integral of the magnetic field component along the LOS $B_{\|}$weighted by the thermal electron density $n_{\mathrm{e}}$ (Jackson 1975):

$\operatorname{RM}(l, b)=\frac{q^{3}}{8 \pi^{2} \epsilon_{0} m_{\mathrm{e}}^{2} c^{3}} \int_{0}^{\infty} \mathrm{d} s n_{\mathrm{e}}(\boldsymbol{r}) B_{\|}(\boldsymbol{r})$,

where $q$ is the elementary charge, $c$ the speed of light in vacuum, $m_{\mathrm{e}}$ is the electron mass, and $\epsilon_{0}$ is the electric constant. The Faraday rotation maps are conveniently expressed in $\left[\mathrm{rad} \mathrm{m}^{-2}\right]$.

Another useful quantity in radioastronomy is the dispersion measure which corresponds to the integrated column density of free electrons and is calculated using:

$\operatorname{DM}(l, b)=\int_{0}^{\infty} \mathrm{d} s n_{\mathrm{e}}(\boldsymbol{r})$.

Dispersion measure is often given in units of $\left[\mathrm{pc} \mathrm{cm}^{-3}\right]$.

\subsection{Free-free emission and absorption}

Free-free emission is produced by free (thermal) electrons scattering off ions in the ionized component of the ISM. This radiation contributes to the unpolarized radio diffuse emission at frequencies above a few $\mathrm{GHz}$ in the Galactic plane where it is produced in the gas layer ionized by radiation from recently formed stars. Free-free transitions of ionized hydrogen are also relevant in absorption of synchrotron emission. This is important at frequencies below $\sim \mathrm{GHz}$ and predominantly along the Galactic plane, but also slightly affects the synchrotron radiation observed at higher frequencies.

For hydrogen gas, the free-free emissivity by neutral plasma (namely when the ion density is equal to the free-electron density: $\left.n_{\mathrm{HII}}=n_{\mathrm{e}}\right)$ at a given observed frequency $v$ is taken as in Longair (2011):

$\epsilon_{v}\left(v, T_{\mathrm{e}}, \boldsymbol{r}\right)=\frac{1}{3 \pi^{2}} \sqrt{\frac{\pi}{6}} \frac{q^{6}}{\epsilon_{0}^{3} c^{3} m_{\mathrm{e}}^{2}} \sqrt{\frac{m_{\mathrm{e}}}{k_{\mathrm{B}} T_{\mathrm{e}}}} g\left(v, T_{\mathrm{e}}\right) n_{\mathrm{e}}(\boldsymbol{r})^{2} \exp \left(-\frac{h v}{k_{\mathrm{B}} T_{\mathrm{e}}}\right)$,

where $k_{\mathrm{B}}$ is Boltzmann constant and $T_{\mathrm{e}} \sim 10^{4} \mathrm{~K}$ is the average temperature of the free electrons in the ISM.

The Gaunt factor at radio wavelengths can be approximated by:

$g\left(v, T_{\mathrm{e}}\right)=\frac{\sqrt{3}}{2 \pi}\left[\ln \left(\frac{128 \epsilon_{0}^{2} k_{\mathrm{B}}^{3} T_{\mathrm{e}}^{3}}{m_{\mathrm{e}} q^{4} v^{2}}\right)-\Gamma^{1 / 2}\right]$,

where $\Gamma \simeq 0.577$. 
From the emissivity, we additionally compute the absorption coefficient $\alpha_{v}^{\mathrm{ff}}$ (i.e., the probability of absorption per unit distance) as in Rybicki \& Lightman (1979):

$\alpha_{v}^{\mathrm{ff}}\left(v, T_{\mathrm{e}}, \boldsymbol{r}\right)=\frac{\epsilon_{v}\left(v, T_{\mathrm{e}}, \boldsymbol{r}\right)}{4 \pi B_{v}\left(v, T_{\mathrm{e}}\right)}$

where $B_{v}$ is the Planck spectrum of the black-body radiation at the temperature $T_{\mathrm{e}}$.

\subsection{Synchrotron emission}

The radio continuum emission of the Milky Way below $\sim 100 \mathrm{GHz}$ mostly originates from the synchrotron process and hence observations of synchrotron intensity and spectral index provide stringent constraints on the interstellar electron spectrum and on the galactic magnetic field models (Jansson \& Farrar 2012; Strong et al. 2011; Di Bernardo et al. 2013; Vittino et al. 2019). At low frequencies, absorption of synchrotron emission by thermal gas (free-free absorption) can become sizeable and must be taken into account.

The emissivity of a population of relativistic electrons is given by Longair (2011):

$\epsilon_{v}(\boldsymbol{r}, v)=\frac{4 \pi}{c} \int \mathrm{d} E \Phi_{\mathrm{e}}(E, \boldsymbol{r}) j_{v}(E, \boldsymbol{r}, v)$,

where $\Phi_{\mathrm{e}}$ is the differential spectrum of relativistic electrons of energy $E$, and $j_{v}$ is the total emissivity of a single electron at a given observational frequency $v$, and depends on the magnetic field projected in the direction perpendicular to the $\operatorname{LOS} B_{\perp}$ as:

$j_{v}=\frac{\sqrt{3} q^{3} B_{\perp}}{8 \pi^{2} \epsilon_{0} c m_{\mathrm{e}}} F\left(\frac{v}{v_{\mathrm{c}}}\right)$.

In Eq. (10) we define the critical frequency $v_{\mathrm{c}}$ as:

$v_{\mathrm{c}}(E, \boldsymbol{r})=\frac{3}{4 \pi} \gamma_{\mathrm{e}}^{2}(E) \frac{q B_{\perp}(\boldsymbol{r})}{m_{\mathrm{e}}}$,

where $\gamma_{\mathrm{e}}$ is the electron Lorentz factor, and $F(x)$ is defined in terms of the Bessel function $K_{5 / 3}$ :

$F(x)=x \int_{x}^{\infty} \mathrm{d} x^{\prime} K_{5 / 3}\left(x^{\prime}\right)$

In order to account for free-free absorption, the LOS integration to compute the synchrotron intensity as a function of the LOS direction becomes:

$I_{v}(v, l, b)=\frac{1}{4 \pi} \int_{0}^{\infty} \mathrm{d} s A_{v}(v, \boldsymbol{r}) \epsilon_{v}(\boldsymbol{r}, v)$,

where $A_{v}=\exp \left(-\int_{0}^{s} \mathrm{~d} s^{\prime} \alpha_{v}^{\mathrm{ff}}\left(\boldsymbol{r}^{\prime}\right)\right)$ is the absorption function which depends on the absorption coefficient $\alpha_{v}^{\mathrm{ff}}$ that we introduce in Sect. 2.2.

As radio emission is often associated with thermal phenomena, the intensity is traditionally stated in terms of the brightness temperature $T_{\mathrm{b}}$ defined as:

$T_{\mathrm{b}}(v, l, b)=\frac{c^{2}}{2 v^{2} k_{\mathrm{B}}} I_{v}(v, l, b)$

\subsection{Inverse Compton}

The inverse Compton (IC) diffuse emission is produced by CR electrons and positrons scattering on low-energy target photons in the Galaxy as UV, optical, infrared, or microwave background photons (the sum of all the fields form the interstellar radiation field (ISRF)). The IC scattering is a major contributor to the diffuse Galactic emission above $\sim \mathrm{MeV}$, and becomes the dominant process at high galactic latitudes because the intensity of gamma radiation from interactions between CRs and gas is greatly reduced (Acero et al. 2016).

The differential cross-section for producing gamma-rays of energy $E_{\gamma}$ by a high-energy electron $E_{\mathrm{e}}$ scattering on a lowenergy photon $E_{\mathrm{ph}}$ is Blumenthal \& Gould (1970):

$$
\begin{aligned}
\frac{\mathrm{d} \sigma_{\mathrm{IC}}}{\mathrm{d} E_{\gamma}}\left(E_{\mathrm{e}}, E_{\mathrm{ph}}, E_{\gamma}\right)= & \frac{3 \sigma_{\mathrm{T}}}{4 E_{\mathrm{ph}} \gamma_{\mathrm{e}}^{2}} \\
& \times\left[2 q \ln q+(1+2 q)(1-q)+\frac{(p q)^{2}(1-q)}{2(1+p q)}\right],
\end{aligned}
$$

where $\sigma_{\mathrm{T}}$ is the Thomson cross-section,

$p=\frac{4 E_{\mathrm{ph}} E_{\mathrm{e}}}{\left(m_{\mathrm{e}} c^{2}\right)^{2}}$,

and

$q=\frac{E_{\gamma}}{4 E_{\mathrm{ph}} \gamma_{\mathrm{e}}^{2}\left(1-\frac{E_{\gamma}}{E_{\mathrm{e}}}\right)} \quad$ in the range $\quad \frac{1}{4 \gamma_{\mathrm{e}}^{2}}<q \leq 1$.

The gamma-ray emissivity is obtained by integrating over the ISRF and over the CR spectrum as follows:

$$
\begin{aligned}
\epsilon_{\mathrm{E}}\left(E_{\gamma}, \boldsymbol{r}\right)= & 4 \pi \int \mathrm{d} E_{\mathrm{ph}} \frac{\mathrm{d} n_{\mathrm{ph}}}{\mathrm{d} E_{\mathrm{ph}}}\left(E_{\mathrm{ph}}, \boldsymbol{r}\right) \\
& \times \int \mathrm{d} E_{\mathrm{e}} \frac{\mathrm{d} \sigma_{\mathrm{IC}}}{\mathrm{d} E_{\gamma}}\left(E_{\mathrm{e}}, E_{\mathrm{ph}}, E_{\gamma}\right) \Phi_{\mathrm{e}}\left(E_{\mathrm{e}}, \boldsymbol{r}\right),
\end{aligned}
$$

where $n_{\mathrm{ph}}$ is the background photon density and $\Phi_{\mathrm{e}}$ is the CR (electrons and positrons) differential flux.

In Eq. (18) we assume isotropic scattering in the sense that both the lepton distribution and the ISRF are assumed isotropic. The effects of anisotropic scattering on the interstellar radiation field (apart from the cosmic microwave background) have been discussed in Moskalenko \& Strong (2000), Orlando \& Strong $(2021)^{9}$.

\subsection{Gamma-ray emission by pion decay}

Gamma rays are produced by the decay of neutral pions created in collisions of CR nuclei with interstellar gas atoms. Neutral pion production is a catastrophic energy loss process for the protons which typically retain about $\sim 20 \%$ of their energy after interaction. The gamma-ray emission can then be used to trace the CR distribution in the Galaxy and to probe its spectrum far from the Solar System (Gaggero et al. 2015b; Acero et al. 2016; Yang et al. 2016).

As the most abundant species in the CR flux are proton and helium, the emissivity from collisions between CR and gas nuclei can be written as:

$$
\begin{aligned}
\epsilon_{\mathrm{E}}\left(E_{\gamma}, \boldsymbol{r}\right)= & 4 \pi n_{\mathrm{H}}(\boldsymbol{r}) \int \mathrm{d} E\left[\Phi_{\mathrm{H}}(E, \boldsymbol{r})\left(\frac{\mathrm{d} \sigma_{\mathrm{p}-\mathrm{p}}}{\mathrm{d} E_{\gamma}}+f_{\mathrm{He}} \frac{\mathrm{d} \sigma_{\mathrm{He}-\mathrm{p}}}{\mathrm{d} E_{\gamma}}\right)\right. \\
& \left.+\Phi_{\mathrm{He}}(E, \boldsymbol{r})\left(\frac{\mathrm{d} \sigma_{\mathrm{p}-\mathrm{He}}}{\mathrm{d} E_{\gamma}}+f_{\mathrm{He}} \frac{\mathrm{d} \sigma_{\mathrm{He}-\mathrm{He}}}{\mathrm{d} E_{\gamma}}\right)\right],
\end{aligned}
$$

9 See also https://gitlab.mpcdf.mpg.de/aws/stellarics 
where $n_{\mathrm{H}}$ is the interstellar hydrogen density, $\Phi_{i}$ is the CR differential flux of the CR species $i$ as a function of the kinetic energy per nucleon $E$, and $\mathrm{d} \sigma_{i-j} / \mathrm{d} E_{\gamma}$ is the differential cross-section of secondary gamma-ray production in $i-j$ interactions. We assume here that the ISM gas is a mixture of hydrogen and helium nuclei with uniform density ratio $f_{\mathrm{He}}=0.1$.

The interstellar hydrogen is predominantly made of neutral (HI) and molecular hydrogen $\left(\mathrm{H}_{2}\right)$. These gas components, especially $\mathrm{H}_{2}$ which is mostly concentrated in dense clouds, follow complex geometrical structures which cannot be directly inferred from observations. In fact, a detailed knowledge of the gas kinematics is required in order to translate spectroscopic data into three-dimensional distributions. Some models for the HI and $\mathrm{H}_{2} 3 \mathrm{D}$ spatial distributions have been developed either under the form of analytical expressions or as tables where the gas density can be read at given positions (Nakanishi \& Sofue 2003; Ferrière et al. 2007; Pohl et al. 2008; Mertsch \& Vittino 2020). These models can be easily used as input in HERMES to compute the gamma-ray emissivity as a function of position and finally the intensity along a given LOS.

An alternative strategy originally devised by the authors of the GALPROP code (Strong et al. 2000, 2009) makes direct use of the column density skymaps derived from radio measurements. Moreover, column density maps have been obtained in the form of "rings" corresponding to different intervals of the galactocentric radius by combing sky surveys of the $21 \mathrm{~cm}$ emission of HI and surveys of $\mathrm{CO}$ spectral lines - tracing $\mathrm{H}_{2}$ - with Galactic rotation curves.

We can then obtain the total gas column density $N_{\mathrm{H}}$ within the $i$ th ring and as a function of $(l, b)$ as:

$N_{\mathrm{H}}^{i}(l, b)=N_{\mathrm{HI}}^{i}(l, b)+2 N_{\mathrm{H}_{2}}^{i}(l, b)=N_{\mathrm{HI}}^{i}(l, b)+2 X_{\mathrm{CO}}^{i} w_{\mathrm{CO}}^{i}(l, b)$,

where $X_{\mathrm{CO}}(r)$ is the $\mathrm{CO}-\mathrm{H}_{2}$ conversion factor and is given in units of $\left[10^{20} \mathrm{~cm}^{-2} \mathrm{~K}^{-1} /\left(\mathrm{km} \mathrm{s}^{-1}\right)\right]$ and $W_{\mathrm{CO}}$ is the velocityintegrated $\mathrm{CO}$ line intensity measured in $\left[\mathrm{K} \mathrm{km} \mathrm{s}^{-1}\right]$.

The observed intensity in a given direction can consequently obtained as the sum of the gas in each ring multiplied by the emissivity averaged over the ring:

$I_{\gamma}\left(l, b, E_{\gamma}\right)=\frac{1}{4 \pi} \sum_{i} N_{\mathrm{H}}^{i}(l, b)\left\langle\epsilon_{\mathrm{E}}\left(\boldsymbol{r}, E_{\gamma}\right)\right\rangle^{i}$,

where

$\left\langle\epsilon_{\mathrm{E}}\left(\boldsymbol{r}, E_{\gamma}\right)\right\rangle^{i}=\frac{\int_{0}^{\infty} \mathrm{d} s \epsilon_{\mathrm{E}}\left(E_{\gamma}, \boldsymbol{r}\right) p_{\mathrm{HI}}(\boldsymbol{r}) \Theta_{\mathrm{in}}^{i}(\boldsymbol{r})}{\int_{0}^{\infty} \mathrm{d} s p_{\mathrm{HI}}(\boldsymbol{r}) \Theta_{\mathrm{in}}^{i}(\boldsymbol{r})}$,

and $p_{\mathrm{HI}}$ is a smooth function that describes the gas profile at the Galactic scale, and $\Theta_{\text {in }}^{j}(\boldsymbol{r})$ is the Theta-function defined as:

$\Theta_{\text {in }}^{j}(\boldsymbol{r})=\left\{\begin{array}{lll}1 & \text { if } & \boldsymbol{r} \text { inside the } i \text { th ring } \\ 0 & \text { if } & \text { elsewhere }\end{array}\right.$

\subsection{Bremsstrahlung}

Relativistic electrons and positrons additionally lose their energy in the Galaxy by Bremsstrahlung: radiation is emitted by a lepton passing through the electric field of a particle in the ISM (electron or nucleus). As for the synchrotron radiation, this mechanism is inversely proportional to the particle mass and is therefore negligible for protons and nuclei.

The differential bremsstrahlung emissivity in the interstellar space is calculated by:

$$
\begin{aligned}
\epsilon_{\mathrm{E}}\left(E_{\gamma}, \boldsymbol{r}\right)=4 \pi n_{\mathrm{H}}(\boldsymbol{r}) \int_{E_{\gamma}}^{\infty} \mathrm{d} E \Phi_{\mathrm{e}}(E, \boldsymbol{r})[ & \frac{\mathrm{d} \sigma_{\mathrm{br}}}{\mathrm{d} E_{\gamma}}\left(\mathrm{H}, E_{\gamma}, E\right) \\
& \left.+f_{\mathrm{He}} \frac{\mathrm{d} \sigma_{\mathrm{br}}}{\mathrm{d} E_{\gamma}}\left(\mathrm{He}, E_{\gamma}, E\right)\right],
\end{aligned}
$$

where $\mathrm{d} \sigma_{\mathrm{br}} / \mathrm{d} E_{\gamma}$ is the differential cross-section for the emission of a photon of energy $E_{\gamma}$ by an electron or a positron with kinetic energy $E$, and we distinguish between the cases for $\mathrm{H}$ and $\mathrm{He}$.

After computing the emissivity, we obtain the intensity as for the pion decay case by summing over the gas rings as in Eq. (21).

\subsection{The attenuation of gamma-rays due to pair production}

Gamma rays in the energy range $E_{\gamma} \gtrsim 10 \mathrm{TeV}$ suffer nonnegligible absorption during their propagation from the emission point to the Earth. The mechanism that generates the absorption is the creation of electron-positron pairs in photon-photon interactions $\gamma \gamma \rightarrow e^{+} e^{-}$, where the target is provided by the ISRF and the CMB photons (Moskalenko et al. 2006).

The cross-section of the process $\gamma \gamma \rightarrow e^{+} e^{-}$is (Vernetto \& Lipari 2016):

$$
\sigma_{\gamma \gamma}=\sigma_{\mathrm{T}} \frac{3}{16}\left(1-\beta^{2}\right)\left[2 \beta\left(\beta^{2}-2\right)+\left(3-\beta^{4}\right) \ln \frac{1+\beta}{1-\beta}\right],
$$

where $\beta=\sqrt{1-\frac{1}{x}}, x=\frac{E_{\gamma} E_{\mathrm{ph}}}{2\left(m_{\mathrm{e}} c^{2}\right)^{2}}(1-\cos \theta)$, and $\theta$ is the angle between the directions of the interacting photons.

The absorption probability per unit path length (or absorption coefficient) for a gamma-ray of energy $E_{\gamma}$ at the space point $\boldsymbol{r}$ can be calculated integrating the cross-section over the energy and angular distributions of the target photons (which we assume to be isotropic):

$$
\begin{aligned}
K_{\mathrm{pp}}\left(E_{\gamma}, \boldsymbol{r}\right)= & 2 \int \mathrm{d} \theta(1-\cos \theta) \\
& \times \int_{E_{\mathrm{ph}}^{\min }}^{\infty} \mathrm{d} E_{\mathrm{ph}} n_{\gamma}\left(E_{\mathrm{ph}}, \boldsymbol{r}\right) \sigma_{\gamma \gamma}\left[\beta\left(E_{\gamma}, E_{\mathrm{ph}}, \theta\right)\right],
\end{aligned}
$$

where $E_{\mathrm{ph}}^{\min }=2 m_{\mathrm{e}}^{2} c^{4} / E_{\gamma}(1-\cos \theta)$.

The optical depth $\tau$ for photons of energy $E_{\gamma}$ traveling over the distance $\Delta s$ is obtained by integrating the absorption coefficient $K$ along the LOS:

$\tau\left(E_{\gamma}, \Delta s\right)=\int_{\Delta s} \mathrm{~d} s K_{\mathrm{pp}}\left(E_{\gamma}, \boldsymbol{r}\right)$.

The pair-production absorption is believed to be relevant only for gamma-rays with energy much larger than $\sim \mathrm{TeV}$ and passing near the Galactic Centre. In these conditions, the Galactic emission is dominated by the $\pi$-decay and therefore the absorption is only implemented for this process.

However, as accounting for the absorption implies numerically evaluating an additional integral along the LOS for each direction and each ring, which can be computationally expensive, the HERMES user can easily turn the calculation of the absorption on or off to speed up the calculation. When the absorption is taken into account, the ring-averaged gamma-ray emissivity is computed as:

$\left\langle\epsilon_{\mathrm{E}}\left(\boldsymbol{r}, E_{\gamma}\right)\right\rangle^{i}=\frac{\int_{0}^{\infty} \mathrm{d} s \epsilon_{\mathrm{E}}\left(E_{\gamma}, \boldsymbol{r}\right) p(\boldsymbol{r}) \exp \left[-\tau\left(E_{\gamma}, \boldsymbol{r}\right)\right] \Theta_{\mathrm{in}}^{i}(\boldsymbol{r})}{\int_{0}^{\infty} \mathrm{d} s p(\boldsymbol{r}) \Theta_{\mathrm{in}}^{i}(\boldsymbol{r})}$,

where $i$ is the index of the gas-ring considered. 


\subsection{Neutrinos from pion decay}

The diffuse gamma-ray emission of the Galaxy due to the hadronic scattering of the CR sea onto the ISM gas via $\pi^{0}$ decay is accompanied by a corresponding neutrino emission via the decay of charged pions and muons. As gamma rays are also produced by leptonic processes, the possible detection of the Galactic diffuse neutrino emission may then offer a better probe of the CR nuclei population. Moreover, as opposed to gamma-rays, neutrinos are unattenuated even in the PeV energy range, offering a unique way to probe the primary CR spectrum in the knee region even in very far and opaque regions of the Galaxy.

To compute the electron and muon neutrino production spectra we follow the same approach as for gamma-rays. We implement the neutrino emissivity in HERMES using the gas-ring model as in Eq. (21). The cross-sections $\mathrm{d} \sigma_{\mathrm{CR}-\mathrm{p}} / \mathrm{d} E_{v}$ are now computed by summing the contribution of CR $-p$ scattering to $v_{\mathrm{e}}$ and $v_{\mu}$ (and their anti-neutrino counterparts). It can be shown (see e.g., Palladino et al. 2020) that neutrino vacuum oscillations on Galactic distances distribute the total flux among all neutrino flavors almost equally.

\subsection{Dark matter annihilations}

If the DM consists of weakly interacting massive particles (WIMPs), an important tool for inferring their properties could be the detection of gamma-rays and neutrinos produced by the annihilation products of DM in our Galaxy. For WIMPs with masses close to the electroweak scale, $m_{\chi} \sim 100 \mathrm{GeV}-1 \mathrm{TeV}$, the annihilation products are typically found in the $\mathrm{GeV}-\mathrm{TeV}$ range.

The gamma-ray emissivity produced in DM annihilations is described by:

$\epsilon_{\mathrm{E}}\left(E_{\gamma}, \boldsymbol{r}\right)=\frac{1}{4 \pi} \frac{1}{2} \frac{\langle\sigma v\rangle}{m_{\chi}^{2}} \frac{\mathrm{d} N}{\mathrm{~d} E_{\gamma}} \rho_{\chi}^{2}(r)$,

where $m_{\chi}$ is the mass of the WIMP, and $\rho_{\chi}$ is the DM density depending only on the distance to the Galactic Centre $r$. The gamma-ray spectrum generated per WIMP annihilation is $\mathrm{d} N / \mathrm{d} E_{\gamma}$, normalized such that its integral over energy is equal to 1 . The factor ' $1 / 2$ ' accounts for the fact that the $D M$ is assumed to be its own antiparticle. The $\langle\sigma v\rangle$ is the WIMP annihilation cross-section multiplied by the relative velocity of the two WIMPs (averaged over the WIMP velocity distribution), and we assume as benchmark value $\langle\sigma v\rangle=3 \times 10^{-26} \mathrm{~cm}^{3} \mathrm{~s}^{-1}$. Similarly, the neutrino emissivity is obtained as in Eq. (29) with the difference that the neutrino spectrum $\mathrm{d} N / \mathrm{d} E_{v}$ has to be computed.

The integral over the LOS determines the angular dependence of the signal and is controlled by the astrophysical distribution of DM. For typical halo models, this is a function of the radial distance, $r$, strongly peaked towards the Galactic Center.

We consider for the DM distribution the generalized Navarro-Frenk-White profile (gNFW) which is described by:

$\rho_{\chi}(r)=\frac{\rho_{s}}{\left(r / r_{s}\right)^{\gamma}\left(1+r / r_{s}\right)^{3-\gamma}}$,

where $\rho_{s}$ is a normalization constant and $r_{s}$ is a characteristic radius below which the profile scales as $r^{-\gamma}$. The value $\gamma=1$ corresponds to the standard NFW profile (Navarro et al. 1995). The profile parameters $\rho_{s}$ and $r_{s}$ can be obtained in terms of the virial mass $M_{\text {vir }}$ and the concentration parameter $c$. The virial mass is the mass contained in the virial radius $R_{\mathrm{vir}}$. This is defined as the radius of the sphere in which the average DM density is equal to 200 times the critical density of the Universe. The concentration parameter is related to this quantity as $c=R_{\mathrm{vir}} /(2-\gamma) r_{s}$.

\section{The code characteristics and structure}

HERMES is designed with two priorities in mind: ease of use and extensibility. These two priorities dictate the code structure and pattern choices. Our first priority led to the building of an optional self-explanatory Python interface to the code thanks to pybind11 (Jakob et al. 2017), by relying on legible functionalstyle programming as far as modern $\mathrm{C}++$ allows, and by an extensive documentation generated from annotated and commented C++ sources using Doxygen (van Heesch 2018) and Sphinx (Brandl 2020).

Our second priority pushed us to adopt a modular code structure. The modularity means that different logical components of the code, which follow the structure of the previously mentioned physical equations, are separated and independent, and they communicate to each other over standardized and comprehensible interfaces. In addition, a modular code is also easier to read and understand; hence, it contributes to the correctness of the code. However, the most essential parts ensuring the correctness of the code are numerous unit tests (Glenford \& Corey Sandler 2011) and an improved static type system (Pierce \& Benjamin 2002; Gao et al. 2017). The unit tests automatically verify if implemented functions and methods return a correct numerical output for a specified input, while the basic $\mathrm{C}++$ static-type system is enhanced with dimensional analysis of physical quantities and formulas based on Barton \& Nackman (1994), so that the dimensional validity of every physical expression is automatically checked by the compiler.

The code draws upon several main designs and toolset choices from CRPROPA ${ }^{10}$ (Alves Batista et al. 2016), a public code for propagating CR particles and inherits magnetic field models, vectors, and grid classes directly from it. However, as the primary function of HERMES significantly differs from CRPROPA, the major part of the code is newly developed. For some pieces, the code follows design choices of GammaSky, a private code which was successfully exploited to compute gamma-ray models of the Galaxy and is now widely used in experimental collaborations (see, e.g., Gaggero et al. 2015b, 2017).

The minimal elements of any computation in HERMES consist of a skymap, which is a HEALPIX ${ }^{11}$-compatible (Górski et al. 2005) container, and an integrator, which performs the LOS calculation for every pixel of the skymap. The user defines the properties of the skymap container, its resolution (or the number of pixels), the frequency or energy if needed, and attaches a compatible integrator of a relevant physical process that has to be computed. The different integrators require various specific additional components to be specified during the initialization phase, such as for instance a gas density model, a magnetic field model, or a CR flux model. Once all required elements are specified, the computation can be initiated. The final results, contained in the skymap, can be saved, for example, to a FITS-format file. The basic HERMES workflow is illustrated in Fig. 1.

It is worth emphasizing that the skymap pixels are mutually independent and integrators are generally stateless ${ }^{12}$, and so the computation process is thread-safe, and therefore the total computation time in general decreases linearly with the number of threads available. The parallelization is implemented using the native $\mathrm{C}++11$ thread library to ensure cross-platform compatibility.

\footnotetext{
10 https://crpropa.github.io

11 https://healpix.jpl .nasa.gov

12 The memory state of integrators is not changed during the computation of a pixel with the exception of the caching mechanism which can be enabled for certain integrators to gain performance in some cases.
} 


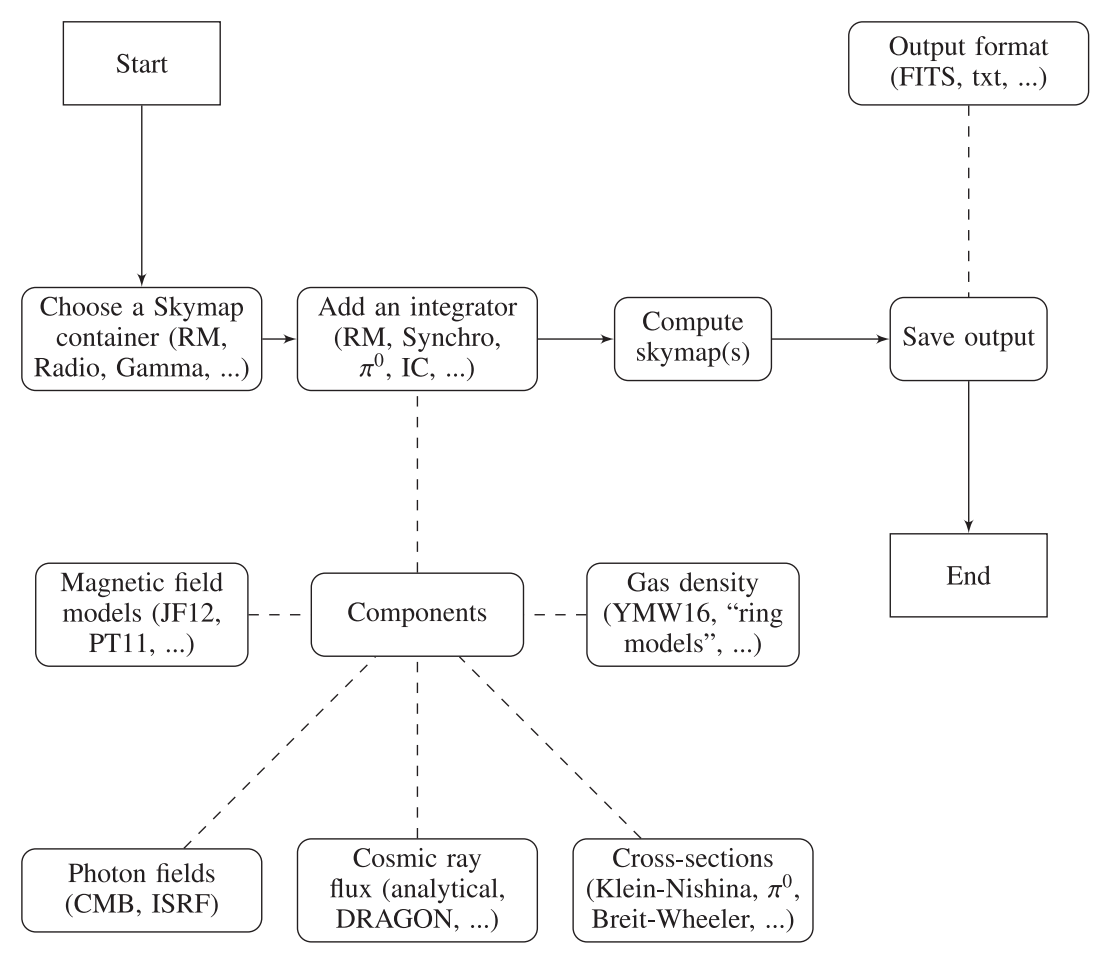

Fig. 1. Flow diagram of basic HERMES usage.

\subsection{Skymap classes}

All skymap classes are initialized first by providing the HEALPIX nside parameter ${ }^{13}$ which determines the total pixel number of a map. A larger number of pixels leads to a higher angular resolution of the skymap.

Several types of skymap classes are available, and they are distinguished based on the physical units of pixels. For example, the rotation measure skymap, RotationMeasureSkymap, stores pixels with the $\left[\mathrm{rad} \mathrm{m}^{-2}\right]$ dimension, the radio skymap defined for a given frequency, RadioSkymap, stores temperature pixels in Kelvins, while the gamma skymap for a given energy, GammaSkymap, stores the differential intensity pixels. Furthermore, for the radio and gamma-ray skymaps, a stacked container abstraction is provided. This stacked container can store multiple skymaps of the same type, ranging in frequency or energy; these are named RadioSkymapRange and GammaSkymapRange.

To each type of skymap, one can attach a specific integrator, map masks, and an output format, either using the class setters or constructors. Integrators are presented in the following section. The map masks are sets of rules specified in the Galactic coordinates and exclude certain regions of the sky from being computed. These so-called unseen pixels are set to $-1.6375 \times 10^{30}$, following the HEALPIX convention. For example, the RectangularWindow class, for two given sets of Galactic latitudes and longitudes, excludes from the computation everything outside of the rectangular window that is stretched between those two coordinate sets. Other provided mask classes, named according to their purpose, are CircularWindow, MaskList, and InvertMask where the last two take mask classes as their arguments; for example, one can use MaskList to combine several rectangular and circular windows into one mask. Finally, by specifying the output format, one determines how the

\footnotetext{
13 The HEALPIX terminology names three related map parameters: the resolution (Res), the number of pixels per side (NSide), and the total pixel number (NPixels). These relate as follows: NSide $=2^{\text {Res }}$ and NPixels $=12 \mathrm{NSide}^{2}$.
}

calculated skymap will be saved after calling its save method. Although the FITS format is provided by default and is generally recommended because it is supported by other software packages such as healpy (Zonca et al. 2019), other save mechanisms can be implemented easily by inheriting the Output abstract class.

\subsection{Integrator classes}

Skymaps only provide the storing facilities for pixels, while integrators evaluate pixels for the directions specified in the traditional spherical coordinates (ISO 80000-2 2009). Each integrator performs the line of sight integration according to the appropriate equations described in Sect. 2, and returns the result in the form of a quantity matching the related skymap class quantity to which the integrator is attached. The integrator classes implemented in HERMES are as follows.

- DispersionMeasureIntegrator is an integrator used in conjunction with DispersionMeasureSkymap and takes a model for the distribution of free (thermal) electrons as an input and returns the dispersion measure in $\left[\mathrm{pc} \mathrm{cm}^{-3}\right]$.

- RotationMeasureIntegrator requires two components: a Galactic field model and a free electron density model following Eq. (4). It returns the rotation measure in $\left[\mathrm{rad} \mathrm{m}^{-2}\right]$.

- SynchroIntegrator takes the magnetic field model and the distribution of CR leptons as input, and returns the synchrotron intensity.

- SynchroAbsorptionIntegrator takes the magnetic field model, the distribution of CR leptons, and the distribution of free electrons as input, and returns the synchrotron intensity, with the free-free absorption taken into account.

- FreeFreeIntegrator takes a model of the distribution of free (thermal) electrons as an input and returns the free-free emissivity (method spectralEmissivity) and absorption coefficient (method absorptionCoefficient).

- PiZeroIntegrator requires as inputs the (hadronic) CR distribution, the (neutral) gas model, and the cross-section 
model, and computes the intensity of the $\pi^{0}$ emission (by summing over the gas rings).

- PiZeroAbsorptionIntegrator takes the ISRF model as additional input with respect to the ones taken by PiZeroIntegrator. The output is the attenuated intensity of the $\pi^{0}$ emission.

- BremsstrahlungIntegrator requires as inputs the (leptonic) cosmic-ray distribution, the (neutral) gas model, and the cross-section model, and computes the bremsstrahlung intensity.

- InverseComptonIntegrator receives as inputs the (leptonic) cosmic-ray distribution, the ISRF model, and the cross-section model, and computes the IC intensity by integrating the emissivity along the LOS.

- DarkMatterIntegrator requires the profile of the Galactic DM halo, the (prompt) gamma-ray spectrum (normalized to one annihilation), and computes the integrated prompt gamma-ray emission.

The examples presented in Sect. 4 make use of these integrators.

\subsection{Astrophysical components as integrator inputs}

Astrophysical fields, particle distribution profiles, interaction cross-sections, and other physical models serve as inputs for the above-described integrators; they define the physical content of any skymap calculation in HERMES.

The code modularity encourages users to modify available components or to adapt and implement new ones. The only requirement for these is to inherit appropriate abstract classes that the given integrator accepts and to implement the corresponding get method(s). In principle, for each look-up, a component can either analytically calculate the required value or extract the value from pre-computed data saved in tables or multidimensional grids where the value in between grid points is interpolated. Both types of look-ups can be delegated to external libraries.

Various tabulation and Cartesian-based grid classes and utilities, such as interpolation techniques or reading and loading functions, are supplied by default to ease the implementations of new components.

In Sect. 4 we introduce several components that we use to present example implementations of the code. Most of these components are either adopted from publicly available codes licensed under the compatible copyleft licences, such as YMW16 and JF12, or newly implemented following original papers. In addition, the models Kamae06 \{Gamma, Neutrino\} depend on an external library, cparamlib ${ }^{14}$, shipped with the code.

\subsection{Code usage}

An important step has been made in designing HERMES following modern standards for modular codes. Different aspects of the simulation (e.g., magnetic fields, CR density, etc.) are separated into modules. Each module is independent from other modules, and therefore each module can be replaced or a new module can be added, making HERMES a flexible framework that can be extended without the need to modify other components. The only requirement for an additional user-provided module is to respect the general module $\mathrm{C}++$ interface, as already explained in Sect. 3.3 for astrophysical components, but applies also for

\footnotetext{
14 The source code of the library with routines written in $\mathrm{C}$ is publicly available at https://github.com/niklask/cparamlib
}

any other module, such as integrators, skymaps, output modules, and skymap masks.

In a user-defined simulation, a sequence of these independent modules is introduced, combined, and wired up. This can be accomplished either through a new $\mathrm{C}++$ program compiled and linked against the HERMES headers and library or via the Python interface imported as pyhermes, a pre-compiled Python module.

A series of examples written in $\mathrm{C}++$, Python, and as Jupiter notebooks illustrate how to use the specific modules in order to compute the maps or the spectra. These are available in a separate repository ${ }^{15}$. Here we present one of the examples provided to compute the $\pi^{0}$ map in order to illustrate the Python interface in more detail.

The example starts with the following initialization commands and general settings:

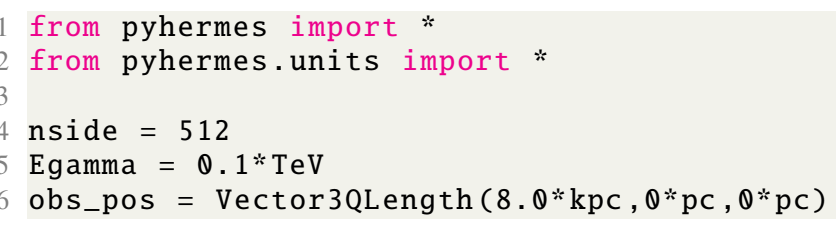

Here we have set a HEALPIX resolution of 512 (corresponding to $\simeq 3.1 \times 10^{6}$ pixels with mean spacing of $\simeq 0.1^{\circ}$ ), a reference energy of $100 \mathrm{GeV}$, and the coordinates associated to the observer (Sun) position in a standard Cartesian grid.

We then specify the astrophysical components:

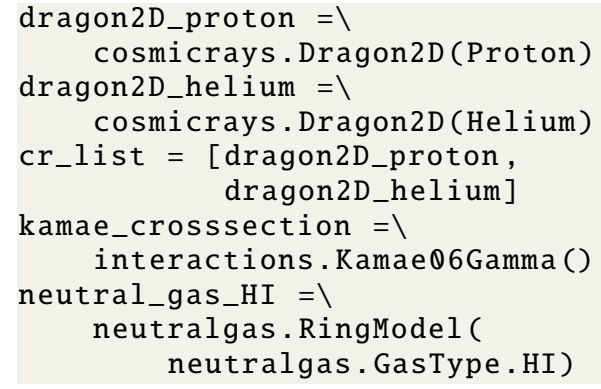

In this block, we have instructed HERMES to load the key components of the computation (see Fig. 1 for a schematic diagram of the different classes involved). These default ingredients are further described in the following section.

In particular we have set:

- the spatial distribution of CR hydrogen and helium nuclei taken (in this case) from the output of a reference DRAGON ${ }^{16}$ run (typically provided in FITS format);

- the cross-section model from Kamae et al. (2006);

- a reference model for the neutral gas distribution (see the following section for more details).

Let us now construct and initialize the appropriate integrator class for the $\pi^{0}$ emission, passing as input parameters the components defined above:

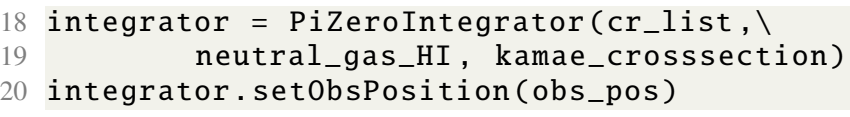

The next integrator command is optional and serves to speed up the calculation. It is discussed in detail in the following section.

21 integrator. setupCacheTable $(100,100,20)$

We proceed with the construction of a new skymap class:

15 http://github.com/cosmicrays/hermes-examples

16 https://github.com/cosmicrays/ 


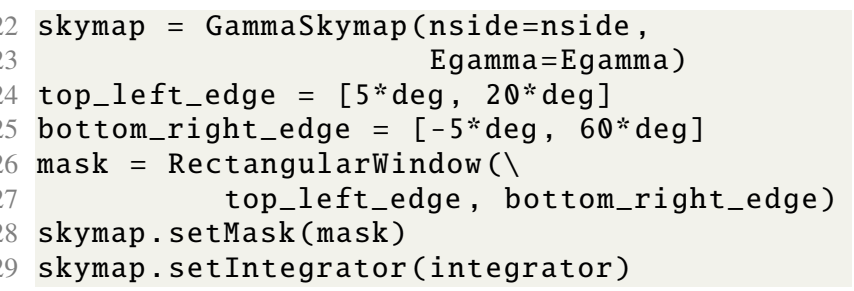

In this block we have instantiated the GammaSkymap class, and attached the already prepared PiZeroIntegrator to it. We have also set a mask associated to a region of interest: a portion of the Galactic plane $\left(20^{\circ}<l<60^{\circ} ;-5^{\circ}<b<5^{\circ}\right)$.

Once all the relevant classes are instantiated and initialized, we call the method that contains the core of the computation:

30 skymap.compute ()

We are now ready to store the result in a numpy array (containing the map in HEALPIX format computed at $100 \mathrm{GeV}$ ), and visualize the map:

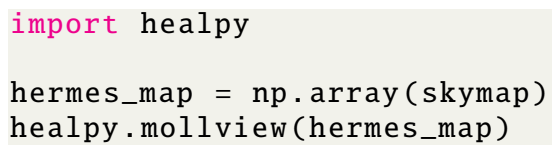

\subsection{Code performance}

HERMES is predominantly a CPU-intensive code because of several nested loops originating from integration procedures listed in Sect. 2. But before discussing the computation time, let us present an overview of the working memory requirements which come, in general, from storing skymap pixels and loading model data. For example, 3.1M double precision floating-point pixels are needed to store one nside $=512$ map. For ranges of skymaps, such as RadioSkymapRange or GammaSkymapRange, this size is multiplied by the number of stacked maps in the range. Consequently, the total consumption of skymap containers is of the order of hundreds of megabytes, depending also on the computer platform used. In comparison, loading some astrophysical components to memory can require considerably more if dense grids are employed, such as in RingModel $(\sim 800 \mathrm{MB})$. The requirements on disk storage of the skymaps and model data is reduced compared to the working memory due to file compression.

The central point of the code performance considerations is the computation time of a HERMES-based program. The computation time is highly sensitive to the execution of the innermost integrand during the LOS integration, especially when triple integrals are used, such as in inverse Compton, Eq. (18), or pion decay, Eq. (19), or quadruple integrals, such as scenarios with the absorption. HERMES automatically employs the multi-threading feature to distribute the LOS integral computation of pixels over all available CPU threads, consequently halving the computation time with respect to the number of threads. The number of threads employed can be controlled with the HERMES_NUM_THREADS environment variable; for example, HERMES_NUM_THREADS=1 will execute the program in a single thread.

Moreover, the computation time can also be reduced with a caching feature (sometimes called memoization), in which the CPU time is traded for the memory consumption. For example, one can pre-compute the innermost integrand in Eq. (18) or Eq. (19) and store the values in a spatial (3D) grid. Then, during the LOS integration, the procedure only accesses and interpolates the pre-computed grid values instead of calculating the integral over and over again. However, in some cases the repeated
Table 1. Average pixel run times for available processes.

\begin{tabular}{llr}
\hline \hline$\#$ & Physical process & $t_{\text {pixel }} / \mathrm{ms}$ \\
\hline 1. & Dispersion measure & 10.3 \\
2. & Rotation measure & 13.7 \\
3. & Synchrotron & 22.7 \\
4. & Free-free & 13.9 \\
5. & Pion decay & 5.4 \\
6. & Bremsstrahlung & 7.1 \\
7. & Inverse Compton & 117.5 \\
8. & Dark matter & 0.3 \\
\hline
\end{tabular}

calculation of the innermost integrand can be faster than the grid look-up and interpolation calls, especially in the multi-threading context where many threads are slowed down by accessing the same shared memory, making the caching feature inefficient.

In HERMES, the cache is calculated before the LOS computation and turned on using the setupCacheTable $\left(N_{X}, N_{Y}\right.$, $N_{Z}$ ) method in gamma-ray-related integrators where $N_{X}, N_{Y}$, $N_{Z}$ are the number of grid points for a galaxy of fixed size $L_{x}, L_{y}=60 \mathrm{kpc}$ and $L_{z}=10 \mathrm{kpc}$ with the GC in the middle of the grid. This feature will be expanded in future versions of HERMES to allow further customization. For optimal performance, the density of the caching grid should match the density of the sparsest grid model used in the innermost integral calculation, such as CR density.

For the example given in the previous section (nside $=512$ ), the measured simulation run time ${ }^{17}$ is $(30.1 \pm 0.3) \mathrm{s}$. The same example, but without caching enabled, that is, with line $21 \mathrm{com}-$ mented out, the measurement gave $(1144 \pm 3) \mathrm{s}$, or approximately 40 times more than with caching. On the other hand, without any skymap mask applied, that is, for the full sky coverage and with caching, the same example is evaluated in $(315 \pm 5) \mathrm{s}$.

An average run time per skymap pixel, $t_{\text {pixel }}$, can be a useful unit with which to estimate the total run time of a simulation simply by evaluating $t_{\text {total }}=N_{\text {total }} / N_{\text {threads }} \times t_{\text {pixel }}$ where $N_{\text {total }}$ is the total number of unmasked pixels which should be computed and $N_{\text {threads }}$ is the number of system threads available. For available processes in realistic scenarios with caching enabled, average run-times per pixel are given in Table 1. The pixel run time of each process significantly depends on the integrator components attached, and not only the integrator implementation, and therefore the code run time can be reduced by optimizing component value retrieval. The results in Table 1 can vary depending on the computer system; they are simply a rough estimate of simulation run times on other systems.

\section{Some relevant applications}

In order to demonstrate the capabilities of HERMES, here we present a set of simulated diffuse emission skymaps and spectra computed using up-to-date models of the relevant Galactic components. The aim of this section is to demonstrate the capability of HERMES and, at the same time, the potential of a multi-wavelength approach, linking radio and gamma-ray data self-consistently to infer the properties of the CR population on Galactic scales.

\footnotetext{
${ }^{17}$ Measured with the hyper fine command-line benchmarking tool on 20 cores $/ 40$ threads at $2.2 \mathrm{GHz}$; HERMES was compiled with GCC 10.2.1.
} 


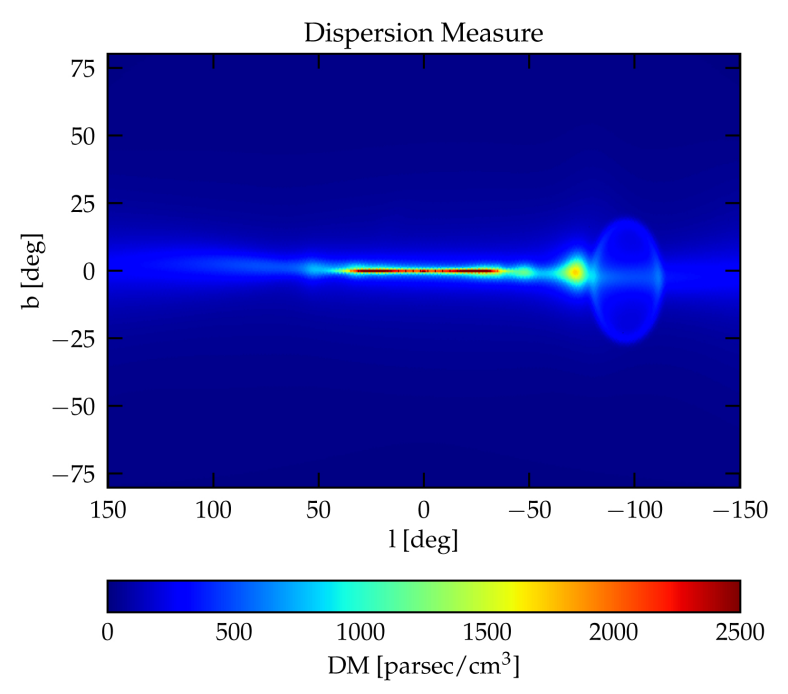

Fig. 2. Cartesian projection in Galactic coordinates of the dispersion measure computed with the free electron model by Yao et al. (2017).

In the following sections, we describe the astrophysical ingredients implemented in a reference setup for HERMES, we provide a comprehensive visualization of the skymaps and spectra at different wavelengths, and we comment on the main features of the resulting emission templates associated to the physical processes discussed above. All input models presented here are optional and can be easily substituted by other models.

For the CR distributions of leptons and nuclei, we adopt the $\mathrm{CR}$ densities obtained by solving the $\mathrm{CR}$ diffusion-loss equation (including re-acceleration) under the assumption of a homogeneous and isotropic diffusion coefficient by means of the DRAGON code (Evoli et al. 2017, 2018). All the assumptions and parameters of the adopted CR model are discussed in Fornieri et al. (2020), where the authors compared their predictions with the most relevant local CR observables (namely: proton, helium, carbon and oxygen flux, boron-to-carbon ratio, low-energy lepton and antiproton fluxes) over a wide energy range (from $\simeq 10 \mathrm{MeV}$ up to $\simeq 1 \mathrm{TeV}$ ).

The map calculations are performed using NSIDE $=256$, corresponding to an angular resolution of $\sim 0.2^{\circ}$.

\subsection{Faraday rotation and dispersion measures of the Galactic magnetic fields}

The full-sky map of Faraday rotation and dispersion measure can be used to derive the properties of the large-scale structure of the Galactic magnetic field and of the electron density.

To simulate these maps, we considered the three-dimensional model by Yao et al. (2017) for the free electrons in the Galaxy. The model features an extended thick disk that represents the so-called warm interstellar medium (WIM), a thin disk representing the Galactic molecular ring, a spiral arm pattern (based on a recent fit to Galactic HII regions), a GC disk, and several local features (including the Gum Nebula, Galactic Loop I, and the Local Bubble). The parameters of this model are fit on a wide set of distance measures and distances of Galactic pulsars.

Concerning the large-scale Galactic magnetic field (GMF) we adopt the model by Jansson \& Farrar (2012), with the updated parameters of Unger \& Farrar (2017) ${ }^{18}$. The model entails a disk component (which follows a spiral-arm pattern), a toroidal halo

18 Available at https://github.com/CRPropa/CRPropa3/blob/ 3.1.6/src/magneticField/JF12Field.cpp component, and an axisymmetric and poloidal out-of-plane component. The model is based on a best fit of a comprehensive set of data, including the WMAP7 maps of synchrotron emission and rotation measures of $4 \times 10^{4}$ extragalactic sources.

The turbulent magnetic field model is computed by a class obtained from CRPROPA (Alves Batista et al. 2016), where the field is implemented following an approach firstly developed by Giacalone \& Jokipii (1994). The field is initially constructed in a discrete $k$-space $\boldsymbol{B}(\boldsymbol{k})$ by drawing for each grid point (in total $\mathrm{N}^{3}$ ) a randomly oriented vector $\boldsymbol{k}$ which satisfies solenoidality, i.e., $\boldsymbol{k}$. $\boldsymbol{B}=0$. The vector amplitude is determined from a turbulent power spectrum $\boldsymbol{B}^{2}(k)=k^{\alpha}$ where the default value $\alpha=-11 / 3$ represents the Kolmogorov spectrum in 3D space. The amplitude is further modulated by a random complex phase. Every $\boldsymbol{B}(\boldsymbol{k})$ that lies outside of the range $\left[k_{\min } \equiv \operatorname{spacing} / L_{\max }, k_{\max } \equiv \operatorname{spacing} / L_{\min }\right]$ is set to zero, where $k_{\min }$ and $k_{\max }$ are the minimum and maximum wavenumbers and spacing is the physical distance between two nearest grid points. In doing so, $L_{\max }$ and $L_{\min }$ are the maximal and minimal physical scale of turbulence and are user-defined quantities. Subsequently, the $k$-field is transformed into real space with a Fast Fourier Transform method provided from the FFTW software package (Frigo \& Johnson 2005). Finally, the grid is normalized at each position to the local value of $B_{\text {rms }}$ given by the magnetic field model of Jansson \& Farrar (2012). For details of the turbulent field implementation see, for example, Appendix B in Dundovic (2018).

Figure 2 shows our full-sky map of the dispersion measure in which the contribution of the Galactic plane is visible, as are several local structures associated with the adopted electron density distribution. In Fig. 3 we visualize the rotation measure sky map and we compare the case with and without the turbulent magnetic field.

\subsection{Radio emissions}

Figure 4 (panel A) shows our the sky map of the simulated synchrotron intensity. We compute the map at the reference frequency of $408 \mathrm{MHz}$, because it is widely covered by high-quality all-sky continuum surveys (see for instance Haslam et al. 1981), and the Galactic synchrotron emission is the dominant contribution. The dominant contribution from the Galactic plane can be clearly identified in the map.

The spatial variation of the spectral slope at that reference frequency is also shown in Fig. 4 (panel B). This slope is directly connected to the slope of the population of CR electrons at a few $\mathrm{GeV}$. A clear trend is the progressive softening towards greater latitudes, as expected when the portion of the leptonic CR population that is probed is located farther away from the regions where most of the acceleration takes place.

The synchrotron spectrum in the sky region (for both full sky and the intermediate-latitude region defined by $10^{\circ}<b<40^{\circ}$ ) is compared in Fig. 5 with the spectrum of free-free emission. We notice in particular that synchrotron emission is the dominant process up to $\simeq 10 \mathrm{GHz}$, the band at which free-free emission, featuring a harder spectrum, starts to take over. The impact of absorption is small. Only at small frequencies (around $1 \mathrm{MHz}$ ) is the effect around 10-20\%.

\subsection{Gamma-ray sky below $1 \mathrm{TeV}$}

In this section we analyze the HERMES predictions for the gamma-ray sky in the GeV-TeV domain. As mentioned already in Sect. 2, the main emission mechanisms in this energy range are $\pi^{0}$ decay, inverse Compton scattering, and bremsstrahlung. 
A. Dundovic et al.: Simulating the Galactic multi-messenger emissions with HERMES
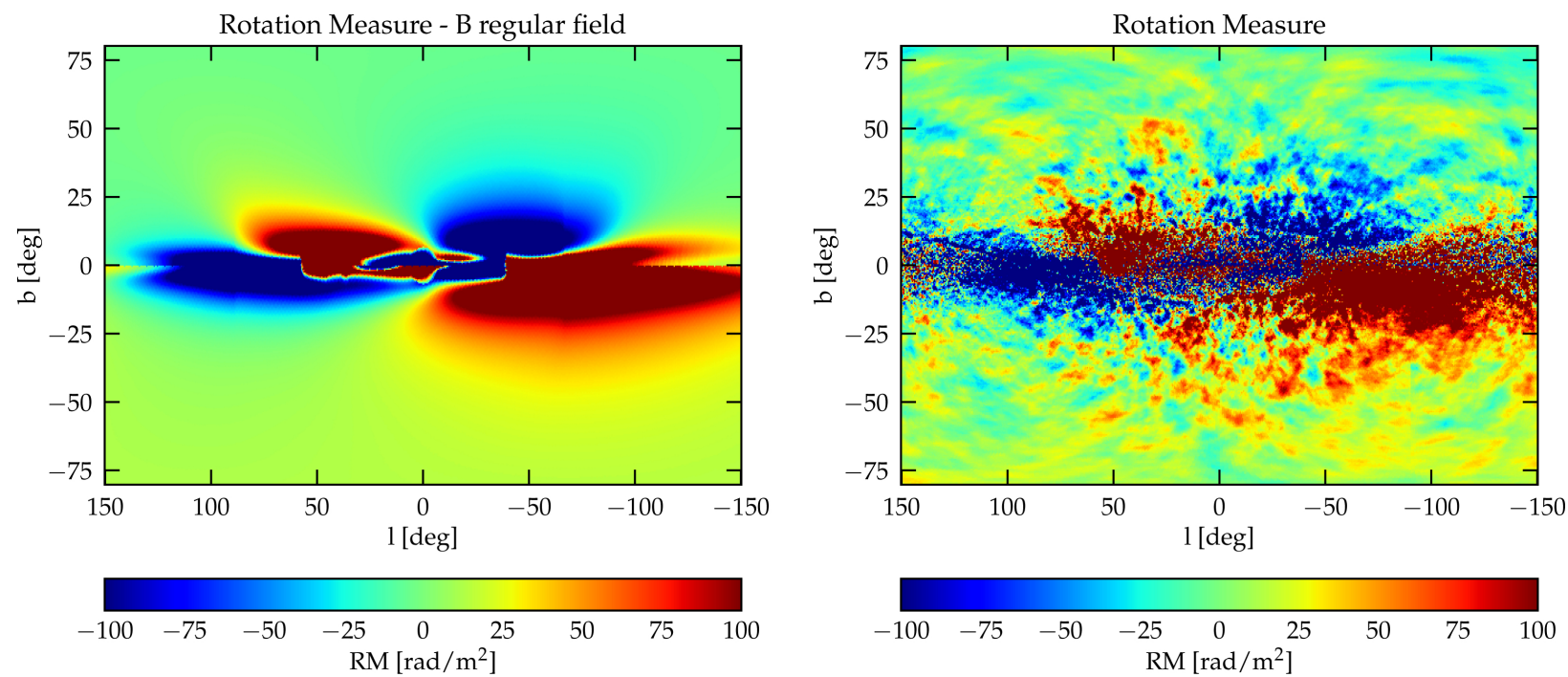

Fig. 3. Cartesian projection in Galactic coordinates of the rotation measure. Left panel: map computed with the large-scale Galactic magnetic field model provided by Jansson \& Farrar (2012). Right panel: computation including a model of the turbulent field computed as detailed in Sect. 4.1.
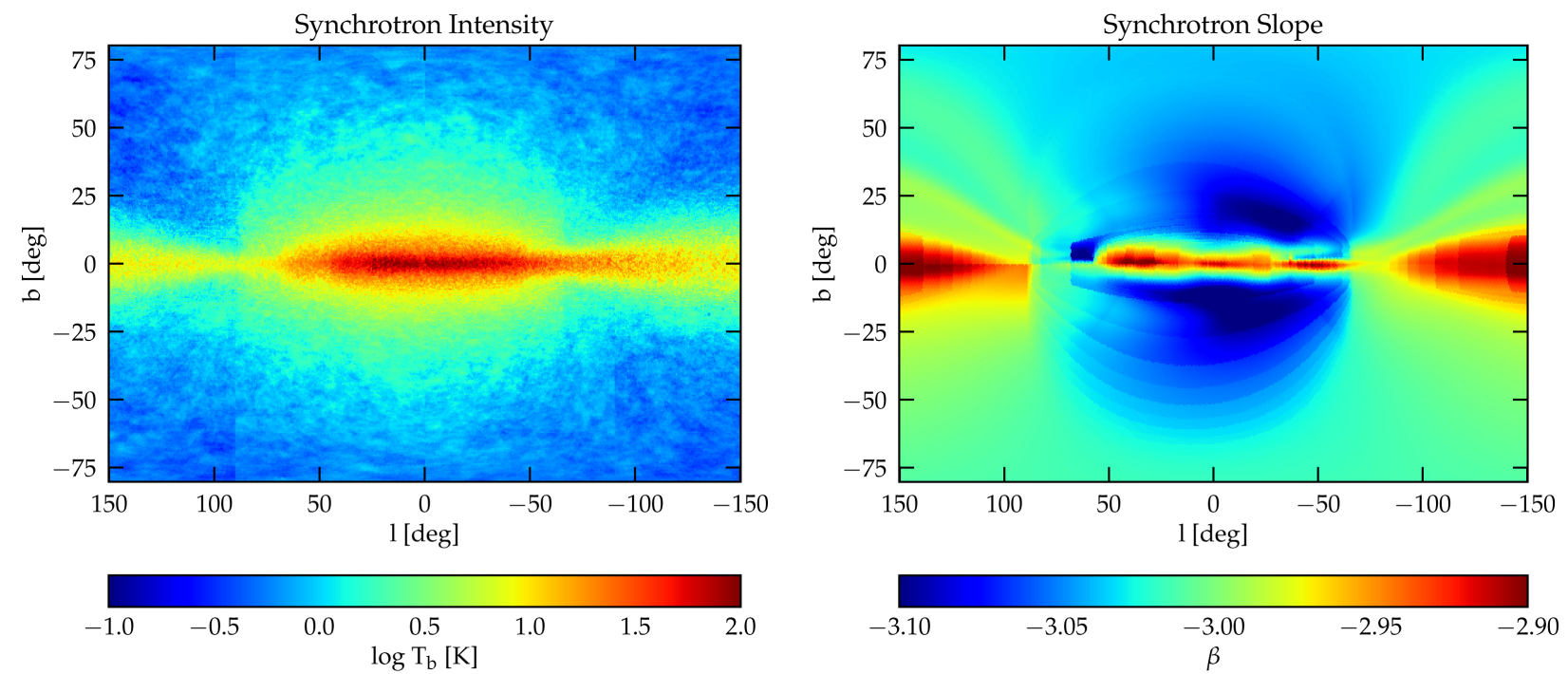

Fig. 4. Cartesian projection in Galactic coordinates of the synchrotron intensity (left panel) and of the synchrotron slope (right panel). Both plots are computed at the frequency of $408 \mathrm{MHz}$.
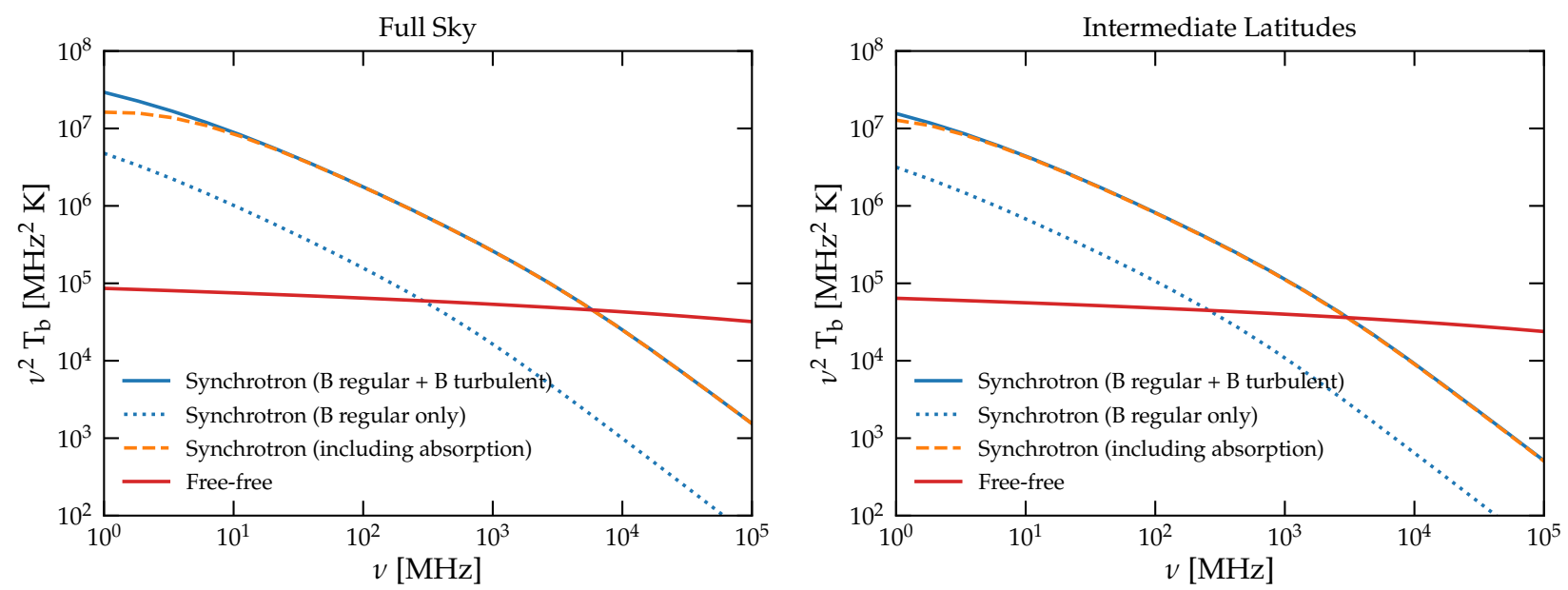

Fig. 5. Integrated spectra associated to the most relevant processes in the radio domain: synchrotron emission (without or with free-free absorption) and free-free emission. Left panel: full-sky integration. Right panel: intermediate-latitude region defined by $10^{\circ}<b<40^{\circ}$. 

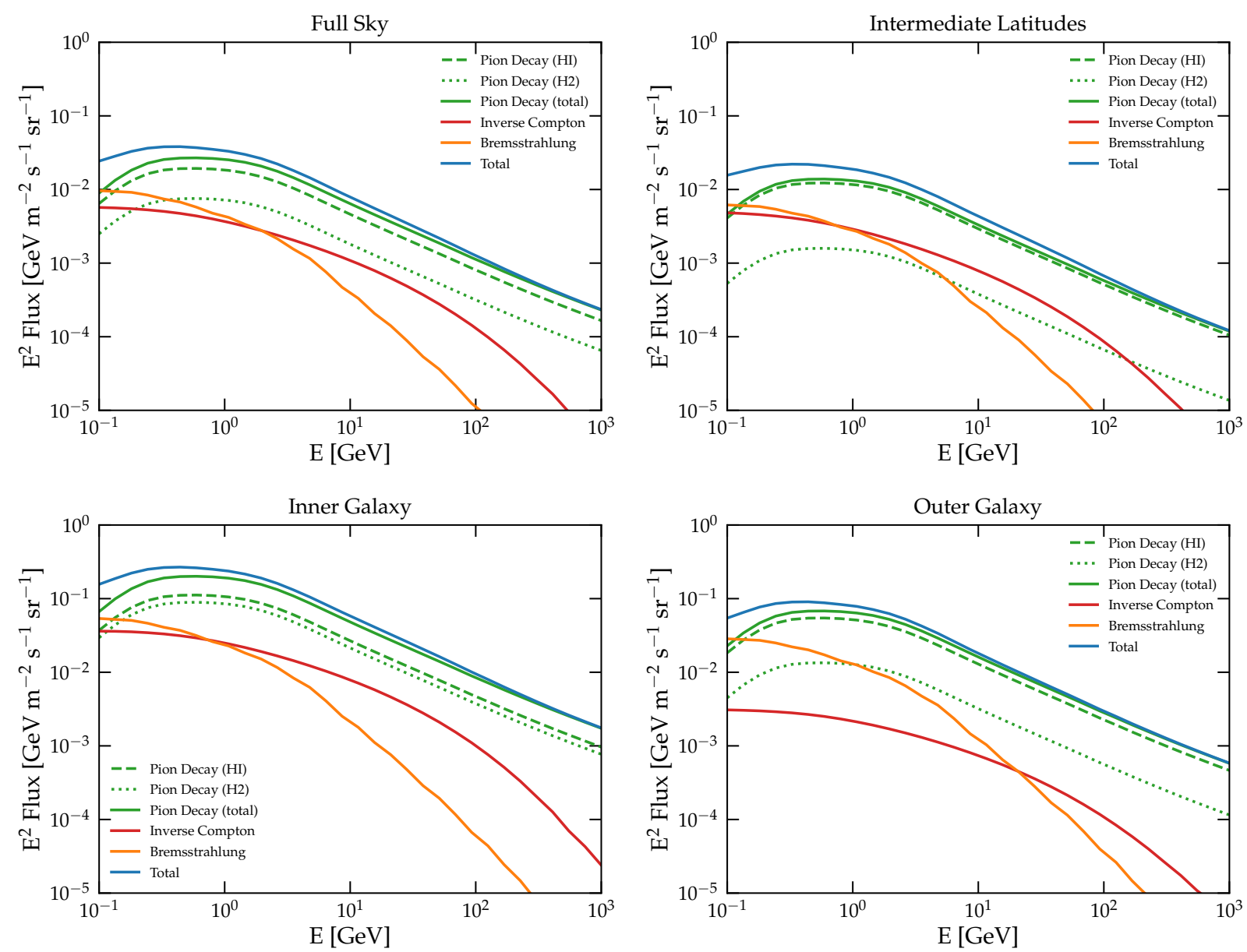

Fig. 6. Gamma-ray spectra extracted from four sky regions: full-sky (top-left), intermediate latitudes $\left(10^{\circ}<|b|<40^{\circ}\right.$, top-right), inner Galaxy $\left(|b|<8^{\circ} \wedge 80^{\circ}<l<280^{\circ}\right.$, bottom-left), and outer Galaxy $\left(|b|<8^{\circ} \wedge l<80^{\circ} \wedge l>280^{\circ}\right.$, bottom-right). The total gamma-ray spectrum (blue solid line) is computed as the sum of three basic emission components: inverse Compton (red solid line), bremsstrahlung (orange solid line), and $\pi^{0}$-decay (green solid line). For the pion decay we show the contributions of HI (green dashed line) and of $\mathrm{H}_{2}$ (green dotted line) separately.

Computation of the emission from $\pi^{0}$ decay is required when modeling the target gas distribution as a set of column density full-sky maps associated to different Galactocentric rings as described in Sect. 2.5. In HERMES, we adopt the recent model developed by Q. Remy of the molecular and atomic interstellar gas (Remy et al., in prep.).

In particular the molecular gas distribution is based on the $115 \mathrm{GHz}$ CfA CO survey in Dame et al. (2001) and Dame \& Thaddeus (2004), which provides a high-resolution $\left(0.125^{\circ}\right.$ spacing $)$ sampling along the Galactic plane, and a slightly less refined sampling $\left(0.25^{\circ}\right)$ at high latitudes $\left(|b|>10^{\circ}\right)$. Concerning the atomic part, the model adopts the HI4PI survey by HI4PI Collaboration (2016) of the 21-cm emission of neutral hydrogen (the angular resolution is $\simeq 0.26^{\circ}$ ), which traces the whole neutral atomic gas, including the cold (CNM) and the warm (WNM) components. The emission from each line of sight is decomposed into 11 different Galactocentric rings by taking into account the Doppler shift from the Galactic rotation. The rotation curve adopted for this purpose is the one derived in Sofue (2015). For the molecular component, two dedicated rings for the inner and outer regions of the central molecular zone are also present.

For this process, the gamma-ray production cross-section is based on the model developed in Kamae et al. (2006). The Kamae formulation entails a parametrization of the gamma- ray, neutrino, and secondary leptons produced by proton-proton interactions in the ISM featuring a logarithmically rising inelastic cross-section, a description of the diffraction dissociation process, and the Feynman scaling violation. The model is validated for CR proton energy up to $\simeq 500 \mathrm{TeV}$.

For the IC calculation, we adopt the ISRF model from Vernetto \& Lipari (2016), which includes three main components: the uniform cosmic microwave background (CMB), and the spatially dependent infrared (radiated by interstellar dust heated by stars) and stellar emissions (see also Evoli et al. 2017).

Finally, the bremsstrahlung component is computed with the same gas distribution as for the calculation of the $\pi^{0}$ emissivity, and with the differential cross-section described in Tsai (1974). Figure 6 shows the resulting spectra in the GeV-TeV range associated to the different emission components in the following regions of interest (ROIs): full sky; inner Galaxy: $-80^{\circ}<l<80^{\circ} ; 8^{\circ}<b<8^{\circ}$; outer Galaxy: $|l|>80^{\circ} ; 8<b<8^{\circ}$; intermediate latitudes: $10^{\circ}<b<40^{\circ}$.

We notice that the emission from neutral pion decay dominates above $\sim 1 \mathrm{GeV}$ in all the ROIs we considered. The main contribution in the inner Galaxy is due to the interactions with the molecular hydrogen, which is mostly concentrated in a relatively narrow strip (scale height $\lesssim 70 \mathrm{pc}$ ) along the Galactic plane, while at intermediate latitudes the atomic component plays the dominant role due to its more extended latitude profile. 

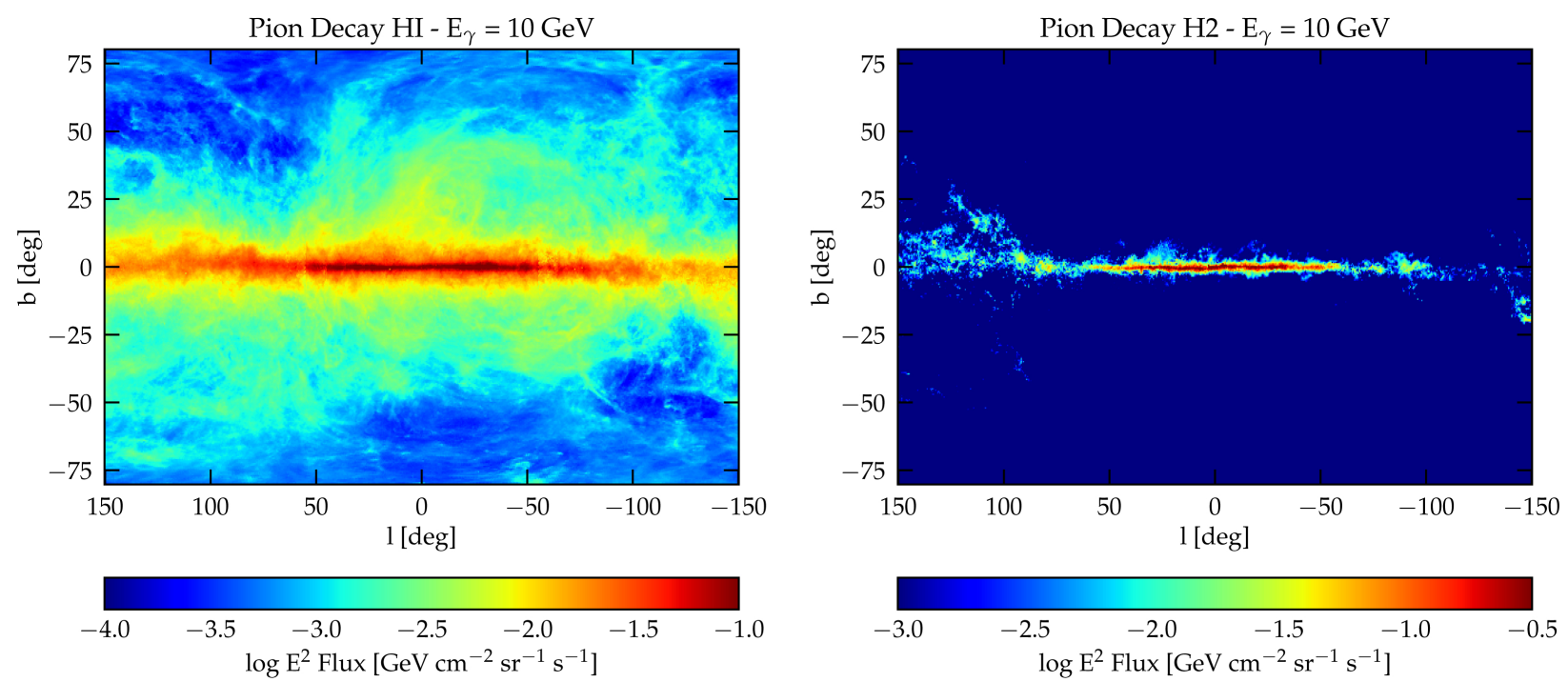

Fig. 7. Gamma-ray sky map at $10 \mathrm{GeV}$. We show the Cartesian projection in Galactic coordinates of the $\pi^{0}$-decay gamma-ray flux at $E_{\gamma}=10 \mathrm{GeV}$. Left panel: gamma-ray emission associated to $\pi^{0}$ production on HI. Right panel: gamma-ray emission associated to $\pi^{0}$ production on $H_{2}$.

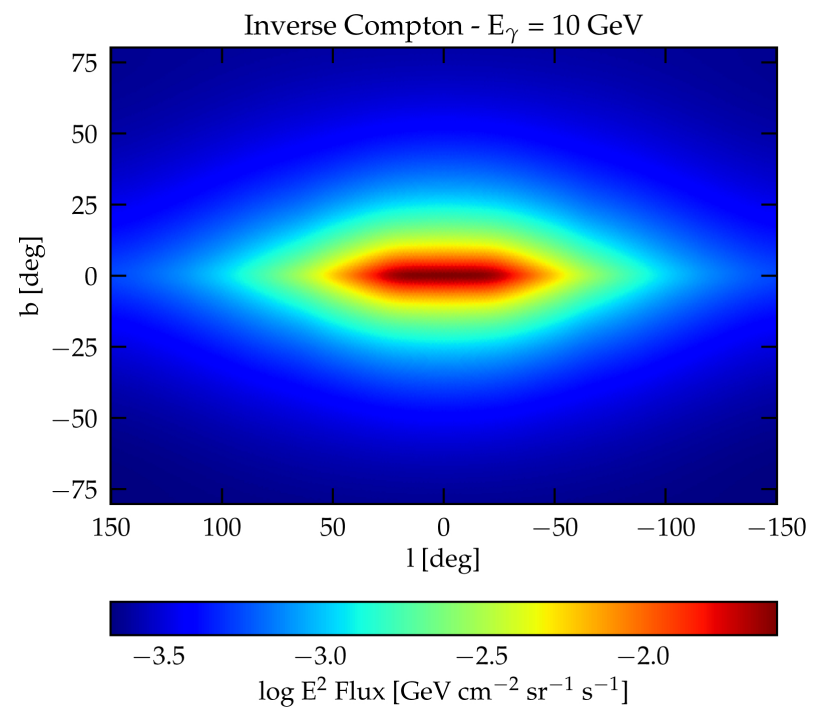

Fig. 8. Cartesian projection in Galactic coordinates of the IC gammaray flux at $E_{\gamma}=10 \mathrm{GeV}$.

The leptonic IC emission is slightly harder in the $1-10 \mathrm{GeV}$ range, and exhibits a clear cutoff at the highest energies. This feature stems from the cutoff in the parent electron population, which is assumed here at $1 \mathrm{TeV}$, while the proton population responsible for the $\pi^{0}$ emission is modeled as an almost featureless power law up to the CR knee (with the only exception being the mild hardening at a rigidity $\simeq 200 \mathrm{GV}$ as identified by the PAMELA and AMS experiments). The IC emission always appears subdominant with respect to the hadronic component, but it becomes more relevant in the mid-latitude regions, because of the larger scale height of the ISRF (compared to both the molecular and atomic gas).

Figures 7 and 8 show the full-sky maps associated to the aforementioned components. The $\pi^{0}$ intensity maps are shown separately for the neutral and the molecular hydrogen contributions. The high level of detail of the hadronic maps reflects the high resolution of the radio observations tracing both molecular and atomic gas. Several patterns can be clearly recognized in the maps. The Galactic plane shines in gamma rays, exhibit- ing a flux that is three orders of magnitude higher than the polar regions; the North Polar Spur, the brightest part of Loop I, is also clearly visible above the plane, similarly to in the radio map. At $l \simeq 70^{\circ}$ along the plane, some small-scale features associated to the Cygnus region are also evident, in particular in the map related to the molecular target. We also notice the different level of clumpiness and spatial extension between the maps of the molecular and the neutral hydrogen, with the latter being more spread out and diffuse along the Milky Way. On the other hand, the IC map appears remarkably smoother, because it reflects the more homogeneous distribution of the target.

The results shown in this section provide a conservative estimate (base model) of the nonthermal emission from the Galaxy, namely from radio frequencies all the way up to multi-TeV gamma rays, based on our current knowledge of the normalization and the spectrum of local CR fluxes, of the distribution of the astrophysical targets (as discussed above), and of the simplest assumptions on CR transport.

A comparison or refitting to actual radio and gamma-ray data is beyond the scope of this paper, and so the model should be considered as the minimal conservative prediction of the nonthermal emission from the Milky Way based on the simplest theoretical arguments and independent from any nonlocal observable.

\subsection{High-energy Gamma-ray sky}

Here we show the capability of HERMES in the context of the multi-TeV energy domain. We adopt the same models for the astrophysical targets as described in the previous section. As far as cross sections are concerned, for the gamma-ray and neutrino production we implement the model described in Kelner \& Aharonian (2008) (with the parameterization of the proton-proton total inelastic cross-section updated as in Kafexhiu et al. 2014).

The features of the code we want to emphasize in this context are: (i) the calculation of gamma-ray absorption which becomes relevant above $\sim 10 \mathrm{TeV}$; (ii) the consistent modeling of different messengers (gamma-rays and neutrinos); and (iii) the calculation of DM-induced gamma-ray and neutrino signals, which is of particular relevance because indirect searches for multi-TeV 

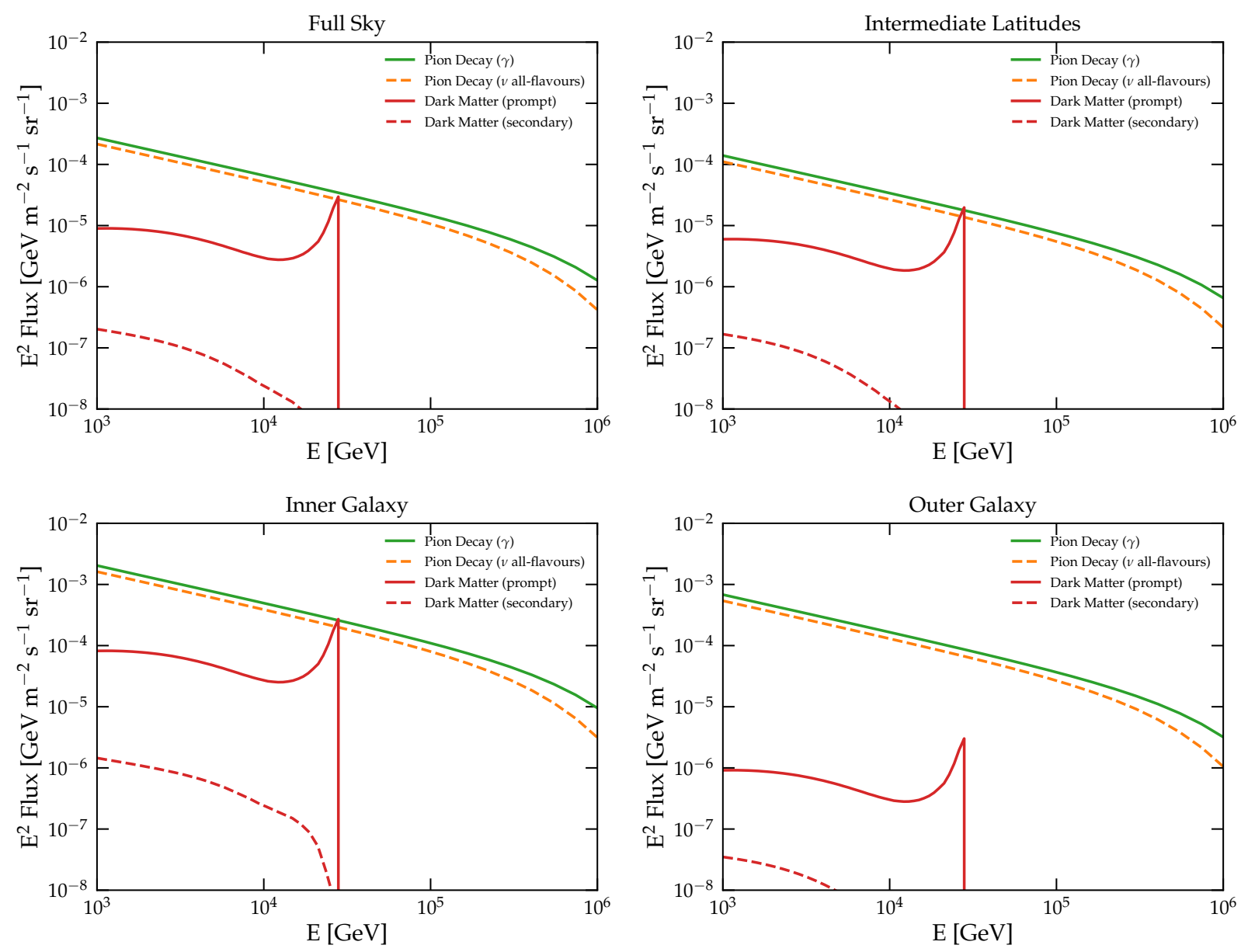

Fig. 9. Spectra extracted from the same four sky regions as in Fig. 6. The gamma-ray spectrum from the $\pi^{0}$-decay (green solid line) is compared with the $v$ spectrum (summed over all flavors) attributable to $\pi^{ \pm}$-decay (orange dashed line). The prompt gamma-ray spectrum expected from DM annihilation in $W^{+} W^{-}$(with mass $M_{\chi}=30 \mathrm{TeV}$ and cross-section $\langle\sigma v\rangle=3 \times 10^{27} \mathrm{~cm}^{2} \mathrm{~s}^{-1}$ ) is shown with a red solid line. The IC from DM secondary leptons is also shown with a red dashed line.

DM candidates will be highly abundant in the coming years (Acharyya et al. 2021).

Figure 9 shows the spectra of gamma-ray (without absorption) and neutrino fluxes as predicted for the pion-decay process, and we compare them with the signal expected for a specific DM candidate. As expected, the neutrino (summed over all flavors) and the gamma-ray spectra exhibit a similar spectral slope and comparable normalization. At the highest energies, the two spectra behave slightly differently because of kinematic effects. The $v / \gamma$ ratio is smaller than 1 at these energies as also found in previous calculations (see for instance the tables reported in Cavasinni et al. 2006).

To illustrate the DM case, we consider a WIMP with $M_{\chi}=$ $30 \mathrm{TeV}$ mainly annihilating into $W^{+} W^{-}$with a cross-section ten times larger than the thermal reference cross-section. Such a combination of mass, annihilation channel, and cross-section is within the reach of the expected performances of CTA. The DM particles are distributed in the Galaxy with a gNFW profile (see Sect. 2.9). The fluxes at production (normalized to one annihilation) for secondary leptons and prompt gamma rays are taken from the public repository ${ }^{19}$ (see Cirelli et al. 2011; Ciafaloni et al. 2011).

In Fig. 9 we compare the signal expected by the prompt emission with the one by secondary emission. The latter is associated

${ }_{19}$ Available at http://wWW . marcocirelli.net/PPPC4DMID .html to the diffuse population of electron+positron pairs produced as a consequence of the $\mathrm{W}$-boson decays. We model the propagation of the secondary particles with DRAGON and compute the corresponding IC emission with HERMES. We notice that, in this energy range, the signal is dominated by the prompt emission. This component features a broad soft continuum due to the fast decay of neutral pions produced by the hadronization of the quark+antiquark pairs in the final state, and a hard feature at high energy with a sharp cutoff associated to final-state radiation. This spectral feature makes the DM signal comparable in intensity with the smooth astrophysical flux at energies close to the DM mass, enabling future searches in the inner Galaxy region with high-resolution observations by upcoming Cherenkov telescopes.

Finally, the impact of gamma-ray absorption is shown for the inner Galaxy in Fig. 10. The difference becomes relevant starting at $\sim 10 \mathrm{TeV}$ and a suppression as large as $\simeq 60 \%$ is reached at $\simeq 100 \mathrm{TeV}$. Including the absorption is therefore relevant for predictions of the very-high-energy flux measured by dedicated experiments such as HAWC and LHAASO.

\section{Conclusions}

We present the HERMES public numerical package which is designed to generate simulated maps and spectra of radio, gamma-ray, and neutrino diffuse emissions originating from the 

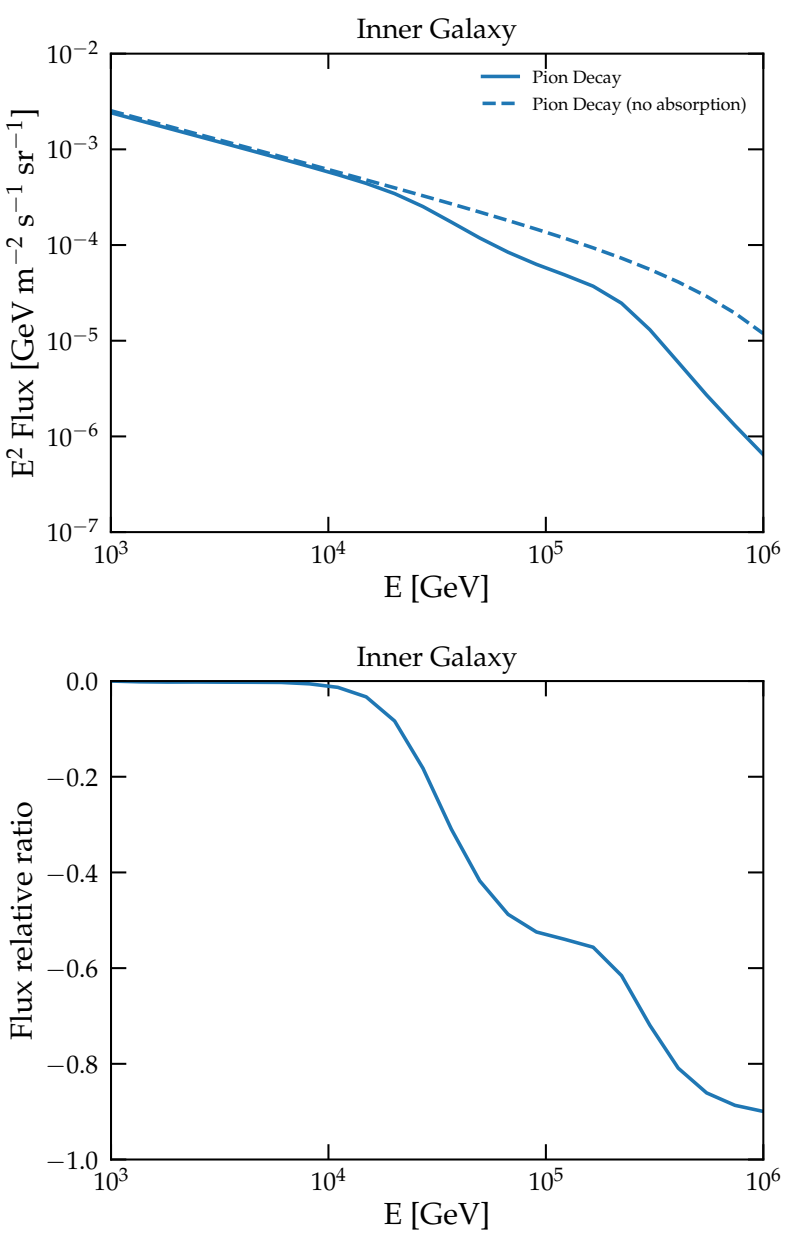

Fig. 10. Top panel: spectrum of the $\pi^{0}$ emission computed for the inner Galaxy ROI (as defined in Fig. 6) together with the case without absorption. Bottom panel: relative ratio of the flux between the case without and with absorption.

interactions of the galactic CR population with the interstellar environment or from DM annihilations and decays in the halo. The physical processes implemented in the code are discussed in Sect. 2.

The modular structure, described in Sect. 3, enables the user to combine independent modules to study multiple use cases and at the same time enables simple updates of all models to meet the current needs of the users.

In order to demonstrate the full capabilities of HERMES we provide example sky maps and spectra (Sect. 4) computed using up-to-date input models from the literature, including the simulated spectra of the prompt and secondary diffuse gammaray emission due to the annihilation of a realistic DM particle physics candidate. A run configuration, including the ingredients to compute the map and the resolution parameters, is fully specified by a Python or $\mathrm{C}++$ script file to be compiled linking HERMES as an external library. To show this, we provide a full and detailed example of a Python script that can be used to compute the gamma-ray sky map of the $\pi^{0}$-decay process. HERMES can be used to constrain the properties of the Galactic CR population and increase our understanding of the radio and gamma-ray diffuse Galactic emission.

Acknowledgements. We are grateful to Pierre Cristofari for reading the text thoroughly and giving helpful feedback. We thank Tess Jaffe, Ralf Kissmann,
Quentin Remy, Andy Strong, Luigi Tibaldo and Silvia Vernetto for useful conversations and insights. We further thank the CRPropa development team for providing a good role model for developing a high-quality $\mathrm{C}++$ astrophysical code and making it available as free software, aside from the fact that HERMES borrows CRPropa's implementation of magnetic fields and abstract vector and grid classes. A.D. acknowledges the P.O.I.N.T. Association, Križevci, Croatia for providing computational resources needed for testing HERMES. This work was funded through Grants ASI/INAF No. 2017-14-H.O. D. Gaggero has received financial support through the Postdoctoral Junior Leader Fellowship Programme from la Caixa Banking Foundation (grant n. LCF/BO/LI18/11630014). D. Gaggero was also supported by the Spanish Agencia Estatal de Investigación through the grants PGC2018-095161-B-I00, IFT Centro de Excelencia Severo Ochoa SEV-2016-0597, and Red Consolider MultiDark FPA2017-90566-REDC. C. Evoli acknowledges the European Commission for support under the H2020MSCA-IF-2016 action, Grant No. 751311 GRAPES 8211 Galactic cosmic RAy Propagation: An Extensive Study.

\section{References}

Acero, F., Ackermann, M., Ajello, M., et al. 2016, ApJS, 223, 26 Acharyya, A., Adam, R., Adams, C., et al. 2021, JCAP, 2021, 057 Adrián-Martínez, S., Ageron, M., Aharonian, F., et al. 2016, J. Phys. G Nucl. Phys., 43, 084001

Aharonian, F. A., \& H.E.S.S. Collaboration 2000, AAS/High Energy Astrophys. Div., 5, 43.11

Ahlers, M., \& Halzen, F. 2018, Progr. Part. Nucl. Phys., 102, 73

Albert, A., André, M., Anghinolfi, M., et al. 2017, Phys. Rev. D, 96, 062001

Albert, A., André, M., Anghinolfi, M., et al. 2018a, ApJ, 853, L7

Albert, A., André, M., Anghinolfi, M., et al. 2018b, ApJ, 868, L20

Alves Batista, R., Dundovic, A., Erdmann, M., et al. 2016, JCAP, 2016, 038

Atwood, W. B., Abdo, A. A., Ackermann, M., et al. 2009, ApJ, 697, 1071

Bai, X., Bi, B. Y., Bi, X. J., et al. 2019, ArXiv e-prints [arXiv:1905.02773]

Barton, J. J., \& Nackman, L. R. 1994, Scientific and Engineering C++: An Introduction with Advanced Techniques and Examples (Addison-Wesley Longman Publishing Co., Inc.)

Beuermann, K., Kanbach, G., \& Berkhuijsen, E. M. 1985, A\&A, 153, 17

Blumenthal, G. R., \& Gould, R. J. 1970, Rev. Mod. Phys., 42, 237

Brandl, G. 2020, Sphinx: Python Documentation Generator, https://www. sphinx-doc.org

Cataldo, M., Pagliaroli, G., Vecchiotti, V., \& Villante, F. L. 2019, JCAP, 2019, 050

Cavasinni, V., Grasso, D., \& Maccione, L. 2006, Astropart. Phys., 26, 41

Cerri, S. S., Gaggero, D., Vittino, A., Evoli, C., \& Grasso, D. 2017, JCAP, 2017, 019

Cherenkov Telescope Array Consortium (Acharya, B. S., et al.) 2019, Science with the Cherenkov Telescope Array, 364

Ciafaloni, P., Comelli, D., Riotto, A., et al. 2011, JCAP, 2011, 019

Cirelli, M., Corcella, G., Hektor, A., et al. 2011, JCAP, 2011, 051

Cortina, J. 2005, Ap\&SS, 297, 245

Dame, T. M., \& Thaddeus, P. 2004, in Milky Way Surveys: The Structure and Evolution of our Galaxy, eds. D. Clemens, R. Shah, \& T. Brainerd, ASP Conf. Ser., 317, 66

Dame, T. M., Hartmann, D., \& Thaddeus, P. 2001, ApJ, 547, 792

Delahaye, T., Fiasson, A., Pohl, M., \& Salati, P. 2011, A\&A, 531, A37

Di Bernardo, G., Evoli, C., Gaggero, D., Grasso, D., \& Maccione, L. 2013, JCAP, 2013, 036

Dundovic, A. 2018, PhD Thesis, Staats-und Universitätsbibliothek Hamburg Carl von Ossietzky, Germany

Evoli, C., Gaggero, D., Vittino, A., et al. 2017, JCAP, 2017, 015

Evoli, C., Gaggero, D., Vittino, A., et al. 2018, JCAP, 2018, 006

Fauvet, L., Macías-Pérez, J. F., Aumont, J., et al. 2011, A\&A, 526, A145

Ferrière, K., Gillard, W., \& Jean, P. 2007, A\&A, 467, 611

Fornieri, O., Gaggero, D., \& Grasso, D. 2020, JCAP, 2020, 009

Frigo, M., \& Johnson, S. G. 2005, Proc. IEEE, 93, 216

Gaggero, D., \& Valli, M. 2018, ArXiv e-prints [arXiv:1802.00636]

Gaggero, D., Urbano, A., Valli, M., \& Ullio, P. 2015a, Phys. Rev. D, 91, 083012

Gaggero, D., Grasso, D., Marinelli, A., Urbano, A., \& Valli, M. 2015b, ApJ, 815, L25

Gaggero, D., Grasso, D., Marinelli, A., Taoso, M., \& Urbano, A. 2017, Phys. Rev. Lett., 119, 031101

Gao, Z., Bird, C., \& Barr, E. T. 2017, Proceedings of the 39th International Conference on Software Engineering, ICSE '17 (Piscataway: IEEE Press), 758

Giacalone, J., \& Jokipii, J. R. 1994, ApJ, 430, L137

Glenford, J. M., \& Corey Sandler, T. B. 2011, The Art of Software Testing, Third Edition (New York: Wiley)

Górski, K. M., Hivon, E., Banday, A. J., et al. 2005, ApJ, 622, 759 
Haslam, C. G. T., Klein, U., Salter, C. J., et al. 1981, A\&A, 100, 209 HI4PI Collaboration (Ben Bekhti, N., et al.) 2016, A\&A, 594, A116

Hunter, S. D., Bertsch, D. L., Catelli, J. R., et al. 1997, ApJ, 481, 205

Hütten, M., Combet, C., \& Maurin, D. 2019, Comput. Phys. Commun., 235, 336

Hutschenreuter, S., Anderson, C. S., Betti, S., et al. 2021, A\&A, submitted [arXiv:2102.01709]

ISO 80000-2 2009, Quantities and Units-Part 2: Mathematical Signs and Symbols to be Used in the Natural Sciences and Technology

Jackson, J. D. 1975, Classical Electrodynamics (New York: Wiley)

Jaffe, T. R., Leahy, J. P., Banday, A. J., et al. 2010, MNRAS, 401, 1013

Jaffe, T. R., Banday, A. J., Leahy, J. P., Leach, S., \& Strong, A. W. 2011 MNRAS, 416, 1152

Jakob, W., Rhinelander, J., \& Moldovan, D. 2017, pybind11 - Seamless Operability Between $\mathrm{C}++11$ and Python, https://github.com/pybind/ pybind 11

Jansson, R., \& Farrar, G. R. 2012, ApJ, 757, 14

Kafexhiu, E., Aharonian, F., Taylor, A. M., \& Vila, G. S. 2014, Phys. Rev. D, 90, 123014

Kamae, T., Karlsson, N., Mizuno, T., Abe, T., \& Koi, T. 2006, ApJ, 647, 692

Kelner, S. R., \& Aharonian, F. A. 2008, Phys. Rev. D, 78, 034013

Kissmann, R. 2014, Astropart. Phys., 55, 37

Kissmann, R., Werner, M., Reimer, O., \& Strong, A. W. 2015, Astropart. Phys., 70,39

Kniffen, D. A., Hartman, R. C., Thompson, D. J., \& Fichtel, C. E. 1973, ApJ 186, L105

Kraushaar, W. L., Clark, G. W., Garmire, G. P., et al. 1972, ApJ, 177, 341

Lebrun, F., Paul, J. A., Bignami, G. F., et al. 1982, A\&A, 107, 390

Longair, M. S. 2011, High Energy Astrophysics (Cambridge: Cambridge University Press)

Men, H., Ferrière, K., \& Han, J. L. 2008, A\&A, 486, 819

Mertsch, P., \& Vittino, A. 2020, ArXiv e-prints [arXiv:2012.15770]

Momcheva, I., \& Tollerud, E. 2015, ArXiv e-prints [arXiv:1507. 03989]

Moskalenko, I. V., \& Strong, A. W. 2000, ApJ, 528, 357

Moskalenko, I. V., Porter, T. A., \& Strong, A. W. 2006, ApJ, 640, L155

Moskalenko, I., Johannesson, G., \& Porter, T. 2019, Int. Cosmic Ray Conf., 36, 111

Nakanishi, H., \& Sofue, Y. 2003, PASJ, 55, 191

Navarro, J. F., Frenk, C. S., \& White, S. D. M. 1995, MNRAS, 275, 56

Neronov, A., \& Semikoz, D. 2016, Astropart. Phys., 75, 60
Orlando, E., \& Strong, A. 2021, JCAP, 2021, 004

Pagliaroli, G., Evoli, C., \& Villante, F. L. 2016, JCAP, 2016, 004

Palladino, A., Spurio, M., \& Vissani, F. 2020, Universe, 6, 30

Pierce, B. C., \& Benjamin, C. 2002, Types and Programming Languages (MIT Press)

Pohl, M., Englmaier, P., \& Bissantz, N. 2008, ApJ, 677, 283

Portegies Zwart, S. 2020, Nat. Astron., 4, 819

Pothast, M., Gaggero, D., Storm, E., \& Weniger, C. 2018, JCAP, 2018, 045

Pshirkov, M. S., Tinyakov, P. G., Kronberg, P. P., \& Newton-McGee, K. J. 2011, ApJ, 738, 192

Recchia, S., Blasi, P., \& Morlino, G. 2016, MNRAS, 462, L88

Rybicki, G. B., \& Lightman, A. P. 1979, Radiative Processes in Astrophysics (New York: Wiley)

Selig, M., Vacca, V., Oppermann, N., \& Enßlin, T. A. 2015, A\&A, 581, A126

Sofue, Y. 2015, PASJ, 67, 75

Strong, A. W., Moskalenko, I. V., \& Reimer, O. 2000, ApJ, 537, 763

Strong, A. W., Moskalenko, I. V., \& Reimer, O. 2004, ApJ, 613, 962

Strong, A. W., Moskalenko, I. V., Porter, T. A., et al. 2009, ArXiv e-prints [arXiv:0907.0559]

Strong, A. W., Orlando, E., \& Jaffe, T. R. 2011, A\&A, 534, A54

Sullivan, G., \& MILAGRO Collaboration 2001, Int. Cosmic Ray Conf., 7, 2773

Sun, X.-H., \& Reich, W. 2010, Res. Astron. Astrophys., 10, 1287

Sun, X. H., Reich, W., Waelkens, A., \& Enßlin, T. A. 2008, A\&A, 477, 573

Tavani, M., Barbiellini, G., Argan, A., et al. 2009, A\&A, 502, 995

Tsai, Y.-S. 1974, Rev. Mod. Phys., 46, 815

Unger, M., \& Farrar, G. R. 2017, Int. Cosmic Ray Conf., 301, 558

van Heesch, D. 2018, Doxygen: Source Code Documentation Generator Tool, https://www.doxygen.nl

Vernetto, S., \& Lipari, P. 2016, Phys. Rev. D, 94, 063009

Vittino, A., Mertsch, P., Gast, H., \& Schael, S. 2019, Phys. Rev. D, 100, 043007

Waelkens, A., Jaffe, T., Reinecke, M., Kitaura, F. S., \& Enßlin, T. A. 2009, A\&A, 495, 697

Wang, J., Jaffe, T. R., Enßlin, T. A., et al. 2020, ApJS, 247, 18

Weekes, T. C., Badran, H., Biller, S. D., et al. 2002, Astropart. Phys., 17, 221

Weltman, A., Bull, P., Camera, S., et al. 2020, PASA, 37, e002

Westerhoff, S. 2014, Adv. Space Res., 53, 1492

Yang, R., Aharonian, F., \& Evoli, C. 2016, Phys. Rev. D, 93, 123007

Yao, J. M., Manchester, R. N., \& Wang, N. 2017, ApJ, 835, 29

Zonca, A., Singer, L., Lenz, D., et al. 2019, J. Open Sour. Softw., 4, 1298 